FUNCTION SPACES XII

BANACH CENTER PUBLICATIONS, VOLUME 119

INSTITUTE OF MATHEMATICS

POLISH ACADEMY OF SCIENCES

WARSZAWA 2019

\title{
THE APPROXIMATION PROPERTY FOR WEIGHTED SPACES OF DIFFERENTIABLE FUNCTIONS
}

\author{
KARSTEN KRUSE \\ Institute of Mathematics, Hamburg University of Technology \\ Am Schwarzenberg-Campus 3, 21073 Hamburg, Germany \\ E-mail: karsten.kruse@tuhh.de
}

\begin{abstract}
We study spaces $\mathcal{C} \mathcal{V}^{k}(\Omega, E)$ of $k$-times continuously partially differentiable functions on an open set $\Omega \subset \mathbb{R}^{d}$ with values in a locally convex Hausdorff space $E$. The space $\mathcal{C} \mathcal{V}^{k}(\Omega, E)$ is given a weighted topology generated by a family of weights $\mathcal{V}^{k}$. For the space $\mathcal{C} \mathcal{V}^{k}(\Omega, E)$ and its subspace $\mathcal{C} \mathcal{V}_{0}^{k}(\Omega, E)$ of functions that vanish at infinity in the weighted topology we try to answer the question whether their elements can be approximated by functions with values in a finite dimensional subspace. We derive sufficient conditions for an affirmative answer to this question using the theory of tensor products.
\end{abstract}

1. Introduction. This paper is dedicated to the following problem: Which vector-valued $k$-times continuously partially differentiable functions can be approximated in a weighted topology by functions with values in a finite dimensional subspace? The answer to this question is closely related to the theory of tensor products and the so-called approximation property. A locally convex Hausdorff space $X$ is said to have (Schwartz') approximation property if the identity $I_{X}$ on $X$ is contained in the closure of $\mathfrak{F}(X)$ in $L_{\kappa}(X)$ where $L_{\kappa}(X)$ denotes the space of continuous linear operators from $X$ to $X$ equipped with the topology of uniform convergence on the absolutely convex compact subsets of $X$ and $\mathfrak{F}(X)$ its subspace of operators with finite rank.

The case $k=0$ is well-studied. In [1], 2] and [3] Bierstedt considered the space $\mathcal{C V}(\Omega, E)$ of all continuous functions $f: \Omega \rightarrow E$ from a completely regular Hausdorff space $\Omega$ to a locally convex Hausdorff space $\left(E,\left(p_{\alpha}\right)_{\alpha \in \mathfrak{A}}\right)$ over a field $\mathbb{K}$ with a topology

2010 Mathematics Subject Classification: Primary 46E40, 46A32; Secondary 46E10.

Key words and phrases: approximation property, tensor product, differentiable, weight, vectorvalued.

The paper is in final form and no version of it will be published elsewhere. 
induced by a Nachbin-family $\mathcal{V}:=\left(\nu_{j}\right)_{j \in J}$ of weights, i.e. the space

$$
\mathcal{C} \mathcal{V}(\Omega, E):=\left\{\left.f \in \mathcal{C}(\Omega, E)|\forall j \in J, \alpha \in \mathfrak{A}:| f\right|_{j, \alpha}<\infty\right\}
$$

where $\mathcal{C}(\Omega, E):=\mathcal{C}^{0}(\Omega, E)$ is the space of continuous functions from $\Omega$ to $E$ and

$$
|f|_{j, \alpha}:=\sup _{x \in \Omega} p_{\alpha}(f(x)) \nu_{j}(x) .
$$

Recall that a family $\mathcal{V}:=\left(\nu_{j}\right)_{j \in J}$ of non-negative functions $\nu_{j}: \Omega \rightarrow[0, \infty)$ is called a Nachbin-family of weights if the functions $\nu_{j}$ are upper semi-continuous and the family is directed, i.e. for every $j, i \in J$ there are $k \in J$ and $C>0$ such that $\max \left(\nu_{i}, \nu_{j}\right) \leq C \nu_{k}$. The notion $\mathcal{U} \leq \mathcal{V}$ for two Nachbin-families means that for every $\mu \in \mathcal{U}$ there is $\nu \in \mathcal{V}$ such that $\mu \leq \nu$.

From the perspective of our problem the space $\mathcal{C} \mathcal{V}(\Omega, E)$ has an interesting topological subspace, namely, the space $\mathcal{C} \mathcal{V}_{0}(\Omega, E)$ consisting of the functions that vanish at infinity when weighted which is given by

$$
\mathcal{C} \mathcal{V}_{0}(\Omega, E):=\left\{f \in \mathcal{C} \mathcal{V}(\Omega, E) \mid \forall \varepsilon>0, j \in J, \alpha \in \mathfrak{A} \exists K \subset \Omega \text { compact }:|f|_{\Omega \backslash K, j, \alpha}<\varepsilon\right\}
$$

where

$$
|f|_{\Omega \backslash K, j, \alpha}:=\sup _{x \in \Omega \backslash K} p_{\alpha}(f(x)) \nu_{j}(x) .
$$

One of the main results from [2] solves our problem for $k=0$, Nachbin-families of weights and involves $k_{\mathbb{R}}$-spaces. A completely regular space $\Omega$ is a $k_{\mathbb{R}}$-space if for any completely regular space $Y$ and any map $f: \Omega \rightarrow Y$ whose restriction to each compact $K \subset \Omega$ is continuous the map is already continuous on $\Omega$ (see [5, (2.3.7) Proposition, p. 22]). Obviously, every locally compact Hausdorff space is a $k_{\mathbb{R}}$-space. Further examples of $k_{\mathbb{R}}$-spaces are metrisable spaces by [13, Proposition 11.5, p. 181] and [8, 3.3.20, 3.3.21 Theorem, p. 152] as well as strong duals of Fréchet-Montel spaces by [9, Proposition 3.27, p. 95] and [16, 4.11 Theorem, p. 39].

Theorem 1.1 ([2, 5.5 Theorem, p. 205-206]). Let E be a locally convex Hausdorff space, $\Omega$ a completely regular Hausdorff space and $\mathcal{V}$ a Nachbin-family on $\Omega$ such that one of the following conditions is satisfied.

(i) $\mathcal{Z}:=\{v: \Omega \rightarrow \mathbb{R} \mid v$ constant, $v \geq 0\} \leq \mathcal{V}$.

(ii) $\mathcal{W}:=\left\{\mu \chi_{K} \mid \mu>0, K \subset \Omega\right.$ compact $\} \leq \mathcal{V}$, where $\chi_{K}: \Omega \rightarrow \mathbb{R}$ is the characteristic function of $K$, and $\Omega$ is a $k_{\mathbb{R}}$-space.

Then the following holds.

a) $\mathcal{C V}_{0}(\Omega) \otimes E$ is dense in $\mathcal{C V}_{0}(\Omega, E)$

b) If $E$ is complete, then

$$
\mathcal{C} \mathcal{V}_{0}(\Omega, E) \cong \mathcal{C} \mathcal{V}_{0}(\Omega) \varepsilon E \cong \mathcal{C} \mathcal{V}_{0}(\Omega) \widehat{\otimes}_{\varepsilon} E
$$

c) $\mathcal{C V}_{0}(\Omega)$ has the approximation property.

Here $\mathcal{C} \mathcal{V}_{0}(\Omega) \otimes E$ stands for the tensor product, $\mathcal{C} \mathcal{V}_{0}(\Omega) \widehat{\otimes}_{\varepsilon} E$ for the completion of the injective tensor product and $\mathcal{C} \mathcal{V}_{0}(\Omega) \varepsilon E:=L_{e}\left(\mathcal{C V}_{0}(\Omega)_{\kappa}^{\prime}, E\right)$ for the $\varepsilon$-product of Schwartz of the spaces $\mathcal{C} \mathcal{V}_{0}(\Omega):=\mathcal{C V}_{0}(\Omega, \mathbb{K})$ and $E$. Part a) gives an affirmative answer to our 
question for the space $\mathcal{C} \mathcal{V}_{0}(\Omega, E)$ since it implies that for every $\varepsilon>0, \alpha \in \mathfrak{A}, j \in J$ and $f \in \mathcal{C V}_{0}(\Omega, E)$ there are $m \in \mathbb{N}, f_{n} \in \mathcal{C} \mathcal{V}_{0}(\Omega)$ and $e_{n} \in E, 1 \leq n \leq m$, such that

$$
\left|f-\sum_{n=1}^{m} f_{n} e_{n}\right|_{j, \alpha}<\varepsilon .
$$

Concerning $\mathcal{C V}(\Omega, E)$, the answer to our question is not that satisfying but still affirmative if we make some restrictions on $E$. If $E$ has the approximation property, then $E \otimes_{\varepsilon} \mathcal{C V}(\Omega)$ is dense in $\operatorname{E\varepsilon \mathcal {CV}}(\Omega)$. Due to the symmetries $\mathcal{C V}(\Omega) \otimes_{\varepsilon} E \cong E \otimes_{\varepsilon} \mathcal{C V}(\Omega)$ and $\mathcal{C V}(\Omega) \varepsilon E \cong$ $\operatorname{E\varepsilon C\mathcal {V}}(\Omega)$, we infer that $\mathcal{C} \mathcal{V}(\Omega) \otimes_{\varepsilon} E$ is dense in $\mathcal{C V}(\Omega) \varepsilon E \cong \mathcal{C} \mathcal{V}(\Omega, E)$ if $E$ is a semi-Montel space with approximation property and $\mathcal{Z} \leq \mathcal{V}$ or $\Omega$ is a $k_{\mathbb{R}^{-}}$space by [3, 2.12 Satz (1), p. 141]. A second condition for an affirmative answer without supposing that $E$ has the approximation property but putting more restrictions on $\mathcal{C V}(\Omega)$ can be found in [3, 2.12 Satz (2), p. 141].

We aim to prove a version of Bierstedt's theorem for spaces of weighted continuously partially differentiable functions. To the best of our knowledge the approximation problem was not considered in a general setting for $k>0$ and open $\Omega \subset \mathbb{R}^{d}$, i.e. to derive sufficient conditions on the weights and the spaces such that the answer is positive. For special cases with $\Omega=\mathbb{R}^{d}$ like the Schwartz space an affirmative answer was already given in e.g. [21, Proposition 9, p. 108] and [21, Théorème 1, p. 111]. For the space of $k$-times continuously partially differentiable functions on open $\Omega \subset \mathbb{R}^{d}$ with the topology of uniform convergence of all partial derivatives up to order $k$ on compact sets a positive answer can be found in e.g. [23, Proposition 44.2, p. 448] and [23. Theorem 44.1, p. 449]. Let us consider for a moment the latter space and the corresponding proof given by Trèves in [23. The space $\mathcal{C}^{k}(\Omega, E)$ of $k$-times continuously partially differentiable functions on a locally compact Hausdorff space $\Omega$ if $k=0$, resp. open $\Omega \subset \mathbb{R}^{d}$ if $k \in \mathbb{N} \cup\{\infty\}$, is equipped with the system of seminorms given by

$$
q_{K, l, \alpha}(f):=\sup _{\substack{x \in K \\ \beta \in \mathbb{N}_{0}^{d},|\beta| \leq l}} p_{\alpha}\left(\partial^{\beta} f(x)\right), \quad f \in \mathcal{C}^{k}(\Omega, E),
$$

for $K \subset \Omega$ compact, $l \in \mathbb{N}_{0}, 0 \leq l \leq k$ if $k<\infty$, and $\alpha \in \mathfrak{A}$. For $E=\mathbb{K}$ we fix the notion $\mathcal{C}^{k}(\Omega):=\mathcal{C}^{k}(\Omega, \mathbb{K})$ and denote by $\mathcal{C}_{c}^{k}(\Omega)$ the space of all functions in $\mathcal{C}^{k}(\Omega)$ having compact support. Trèves' affirmative answer to our question has the following form.

Theorem 1.2 ([23, Proposition 44.2, p. 448] and [23, Theorem 44.1, p. 449]). Let E be a locally convex Hausdorff space, $k \in \mathbb{N}_{0} \cup\{\infty\}$ and $\Omega$ a locally compact Hausdorff space if $k=0$, resp. an open subset of $\mathbb{R}^{d}$ if $k>0$. Then the following is true.

a) $\mathcal{C}_{c}^{0}(\Omega) \otimes E$ is dense in $\mathcal{C}^{0}(\Omega, E)$.

b) $\mathcal{C}_{c}^{\infty}(\Omega) \otimes E$ is dense in $\mathcal{C}^{k}(\Omega, E)$.

c) If $E$ is complete, then

$$
\mathcal{C}^{k}(\Omega, E) \cong \mathcal{C}^{k}(\Omega) \widehat{\otimes}_{\varepsilon} E .
$$

We observe that $\mathcal{C} \mathcal{W}(\Omega, E)=\mathcal{C W}_{0}(\Omega, E)=\mathcal{C}^{0}(\Omega, E)$ equipped with the usual topology of uniform convergence on compact subsets of $\Omega$ which means that Theorem 1.1 contains the case $k=0$ of the preceding theorem since locally compact Hausdorff spaces 
are $k_{\mathbb{R}}$-spaces. The proofs of Theorem 1.1 a) and Theorem 1.2 a) are done by using different partitions of unity, the first uses the partition of unity from [20, 23, Lemma 2, p. 71] and the second the one from [4, Chap. IX, §4.3, Corollary, p. 186]. The key idea for the proof of Theorem $1.2 \mathrm{~b}$ ) is an approximation in three steps relying on part a) and convolution. First, for every $f \in \mathcal{C}^{k}(\Omega, E)$ there is an approximation $\tilde{f} \in \mathcal{C}_{c}^{k}(\Omega, E)$ of $f$ by multiplication of $f$ with a suitable cut-off function. Second, for every $\widetilde{f} \in \mathcal{C}_{c}^{k}(\Omega, E)$ the convolution $\tilde{f} * \rho_{n}$ of $\tilde{f}$ with a sequence $\left(\rho_{n}\right)$ of mollifiers in $\mathcal{C}_{c}^{\infty}(\Omega)$ converges to $\widetilde{f}$ in $\mathcal{C}^{k}(\Omega, \widehat{E})$ where $\widehat{E}$ denotes the completion of $E$ (approximation by regularisation). Third, for every $\tilde{f} \in \mathcal{C}_{c}^{k}(\Omega, E)$ there is an approximation $g \in \mathcal{C}_{c}^{0}(\Omega) \otimes E$ in the topology of $\mathcal{C}^{0}(\Omega, E)$ by part a). Using the properties of the convolution, one gets that $g * \rho_{n} \in \mathcal{C}_{c}^{\infty}(\Omega) \otimes E$ and approximates $\tilde{f} * \rho_{n}$ for $n$ large enough in $\mathcal{C}^{k}(\Omega, \widehat{E})$ which itself is identical to the completion of $\mathcal{C}^{k}(\Omega, E)$.

The outline of our paper is along the lines of Trèves' proof. After introducing some notation and preliminaries in Section 2, we define the weighted spaces $\mathcal{C} \mathcal{V}^{k}(\Omega, E)$ and $\mathcal{C V}_{0}^{k}(\Omega, E)$ in Section 3 and show that they are complete if the family of weights $\mathcal{V}^{k}$ is locally bounded away from zero (see Definition 3.6). Then we treat their relation to the space $\mathcal{C}_{c}^{k}(\Omega, E)$ of functions in $\mathcal{C}^{k}(\Omega, E)$ with compact support where the condition of local boundedness of a family of weights comes into play (see Definition 3.8. We formulate a cut-off criterion (see Definition 3.10 which is a sufficient condition for the density of $\mathcal{C}_{c}^{k}(\Omega, E)$ in $\mathcal{C V}_{0}^{k}(\Omega, E)$ for locally bounded $\mathcal{V}^{k}$. We close the third section with the relation between tensor products and our problem on finite dimensional approximation. In Section 4 we define the convolution $f * g$ of $f \in \mathcal{C}^{k}\left(\mathbb{R}^{d}, E\right)$ and $g \in \mathcal{C}^{n}\left(\mathbb{R}^{d}\right)$ when one of them is compactly supported and prove an approximation by regularisation result. In the last section we verify the corresponding part a) of Theorem 1.2 for $\mathcal{C V}_{0}^{0}(\Omega, E)$ with locally compact $\Omega$ where we adapt the proof of Theorem 1.1 a) in a way that we can use the partition of unity from [4, Chap. IX, §4.3, Corollary, p. 186] instead and weaken the condition of upper semi-continuity of the weights to being locally bounded and locally bounded away from zero. Then we mix all ingredients to get our main Theorem 5.2 which is a version of Theorem 1.1 and 1.2 for barrelled $\mathcal{C} \mathcal{V}_{0}^{k}(\Omega)$ with a family of weights $\mathcal{V}^{k}$ being locally bounded and locally bounded away from zero if $\mathcal{C} \mathcal{V}_{0}^{k}(\Omega, E)$ fulfils the cut-off criterion.

2. Notation and preliminaries. We set $\mathbb{N}_{\infty}:=\mathbb{N} \cup\{\infty\}$ and $\mathbb{N}_{0, \infty}:=\mathbb{N}_{0} \cup\{\infty\}$. For $k \in \mathbb{N}_{0, \infty}$ we use the notation $\langle k\rangle:=\left\{n \in \mathbb{N}_{0} \mid 0 \leq n \leq k\right\}$ if $k \neq \infty$ and $\langle k\rangle:=\mathbb{N}_{0}$ if $k=\infty$. We equip the spaces $\mathbb{R}^{d}, d \in \mathbb{N}$, and $\mathbb{C}$ with the usual Euclidean norm $|\cdot|$, write $\bar{M}$ for the closure of a subset $M \subset \mathbb{R}^{d}$ and denote by $\mathbb{B}_{r}(x):=\left\{w \in \mathbb{R}^{d}|| w-x \mid<r\right\}$ the ball around $x \in \mathbb{R}^{d}$ with radius $r>0$.

By $E$ we always denote a non-trivial locally convex Hausdorff space, in short lcHs, over the field $\mathbb{K}=\mathbb{R}$ or $\mathbb{C}$ equipped with a directed fundamental system of seminorms $\left(p_{\alpha}\right)_{\alpha \in \mathfrak{A}}$. If $E=\mathbb{K}$, then we set $\left(p_{\alpha}\right)_{\alpha \in \mathfrak{A}}:=\{|\cdot|\}$. Further, we denote by $\widehat{E}$ the completion of a locally convex Hausdorff space $E$. For details on the theory of locally convex spaces see [10, 14] or [18]. 
A function $f: \Omega \rightarrow E$ on an open set $\Omega \subset \mathbb{R}^{d}$ to a locally convex Hausdorff space $E$ is called continuously partially differentiable $\left(f\right.$ is $\left.\mathcal{C}^{1}\right)$ if for the $n$-th unit vector $e_{n} \in \mathbb{R}^{d}$ the limit

$$
\left(\partial^{e_{n}}\right) f(x):=\left(\partial^{e_{n}}\right)^{E} f(x):=\left(\partial_{x_{n}}\right)^{E} f(x):=\lim _{\substack{h \rightarrow 0 \\ h \in \mathbb{R}, h \neq 0}} \frac{f\left(x+h e_{n}\right)-f(x)}{h}
$$

exists in $E$ for every $x \in \Omega$ and $\partial^{e_{n}} f$ is continuous on $\Omega\left(\partial^{e_{n}} f\right.$ is $\left.\mathcal{C}^{0}\right)$ for every $1 \leq n \leq d$. For $k \in \mathbb{N}$ a function $f$ is said to be $k$-times continuously partially differentiable $\left(f\right.$ is $\left.\mathcal{C}^{k}\right)$ if $f$ is $\mathcal{C}^{1}$ and all its first partial derivatives are $\mathcal{C}^{k-1}$. A function $f$ is called infinitely continuously partially differentiable $\left(f\right.$ is $\left.\mathcal{C}^{\infty}\right)$ if $f$ is $\mathcal{C}^{k}$ for every $k \in \mathbb{N}$. For $k \in \mathbb{N}_{\infty}$ the linear space of all functions $f: \Omega \rightarrow E$ which are $\mathcal{C}^{k}$ is denoted by $\mathcal{C}^{k}(\Omega, E)$. Its subspace of functions with compact support is written as $\mathcal{C}_{c}^{k}(\Omega, E)$ where we denote the support of $f \in \mathcal{C}^{k}(\Omega, E)$ by $\operatorname{supp} f$.

Let $f \in \mathcal{C}^{k}(\Omega, E)$. For $\beta \in \mathbb{N}_{0}^{d}$ with $|\beta|:=\sum_{n=1}^{d} \beta_{n} \leq k$ we set $\partial^{\beta_{n}} f:=\left(\partial^{\beta_{n}}\right)^{E} f:=f$ if $\beta_{n}=0$, and

$$
\partial^{\beta_{n}} f:=\left(\partial^{\beta_{n}}\right)^{E} f:=\underbrace{\left(\partial^{e_{n}}\right)^{E} \cdots\left(\partial^{e_{n}}\right)^{E}}_{\beta_{n} \text {-times }} f
$$

if $\beta_{n} \neq 0$ as well as

$$
\partial^{\beta} f:=\left(\partial^{\beta}\right)^{E} f:=\partial^{\beta_{1}} \cdots \partial^{\beta_{d}} f .
$$

Due to the vector-valued version of Schwarz' theorem $\partial^{\beta} f$ is independent of the order of the partial derivatives on the right-hand side and we call $|\beta|$ the order of differentiation. Further, we observe that $e^{\prime} \circ f \in \mathcal{C}^{k}(\Omega)$ and $\left(\partial^{\beta}\right)^{\mathbb{K}}\left(e^{\prime} \circ f\right)=e^{\prime} \circ\left(\partial^{\beta}\right)^{E} f$ for every $e^{\prime} \in E^{\prime}$, $f \in \mathcal{C}^{k}(\Omega, E)$ and $|\beta| \leq k$.

By $L(F, E)$ we denote the space of continuous linear operators from $F$ to $E$ where $F$ and $E$ are locally convex Hausdorff spaces. If $E=\mathbb{K}$, we just write $F^{\prime}:=L(F, \mathbb{K})$ for the dual space. If $F$ and $E$ are (linearly topologically) isomorphic, we write $F \cong E$. The so-called $\varepsilon$-product of Schwartz is defined by

$$
F \varepsilon E:=L_{e}\left(F_{\kappa}^{\prime}, E\right)
$$

where $F^{\prime}$ is equipped with the topology of uniform convergence on absolutely convex compact subsets of $F$ and $L\left(F_{\kappa}^{\prime}, E\right)$ is equipped with the topology of uniform convergence on equicontinuous subsets of $F^{\prime}$ (see [22, Chap. I, §1, Définition, p. 18]). It is symmetric which means that $F \varepsilon E \cong E \varepsilon F$ and in the literature the definition of the $\varepsilon$-product is sometimes done the other way around, i.e. $E \varepsilon F$ is defined by the right-hand side of (2). We write $F \widehat{\otimes}_{\varepsilon} E$ for the completion of the injective tensor product $F \otimes_{\varepsilon} E$ and denote by $\mathfrak{F}(E)$ the space of linear operators from $E$ to $E$ with finite rank. We recall from the introduction that a locally convex Hausdorff space $E$ is said to have (Schwartz') approximation property if the identity $I_{E}$ on $E$ is contained in the closure of $\mathfrak{F}(E)$ in $L_{\kappa}(E):=L_{\kappa}(E, E)$ which is equipped with the topology of uniform convergence on the absolutely convex compact subsets of $E$. The space $E$ has the approximation property if and only if $E \otimes F$ is dense in $E \varepsilon F$ for every locally convex Hausdorff space (every Banach space) $F$ by [15, Satz 10.17, p. 250]. For more information on the theory of $\varepsilon$-products and tensor products see [6], [14] and [15]. 
3. Weighted vector-valued differentiable functions and the $\varepsilon$-product. In this section we introduce the spaces $\mathcal{C V}^{k}(\Omega, E)$ and $\mathcal{C V}_{0}^{k}(\Omega, E)$ we want to consider. Then we turn to the question of completeness of $\mathcal{C V}^{k}(\Omega, E)$ and $\mathcal{C} \mathcal{V}_{0}^{k}(\Omega, E)$ and when $\mathcal{C}_{c}^{k}(\Omega, E)$ is dense in the latter space. At the end of this section we describe their connection to the $\varepsilon$-product and the (completion of the) injective tensor product and derive sufficient conditions such that they coincide.

Definition 3.1 (weight). Let $k \in \mathbb{N}_{0, \infty}$. We say that $\mathcal{V}^{k}:=\left(\nu_{j, l}\right)_{j \in J, l \in\langle k\rangle}$ is a (directed) family of weights on a locally compact Hausdorff space $\Omega$ if $\nu_{j, l}: \Omega \rightarrow[0, \infty)$ for every $j \in J, l \in\langle k\rangle$ and

$$
\forall j_{1}, j_{2} \in J, l_{1}, l_{2} \in\langle k\rangle \exists j_{3} \in J, l_{3} \in\langle k\rangle, C>0 \forall i \in\{1,2\}: \nu_{j_{i}, l_{i}} \leq C \nu_{j_{3}, l_{3}}
$$

as well as

$$
\forall l \in\langle k\rangle, x \in \Omega \exists j \in J: 0<\nu_{j, l}(x) .
$$

Definition 3.2. For $k \in \mathbb{N}_{0, \infty}$ and a (directed) family $\mathcal{V}^{k}:=\left(\nu_{j, l}\right)_{j \in J, l \in\langle k\rangle}$ of weights on a locally compact Hausdorff space $\Omega$ if $k=0$ or an open set $\Omega \subset \mathbb{R}^{d}$ if $k \in \mathbb{N}_{\infty}$ we define the space of weighted continuous, resp. $k$-times continuously partially differentiable, functions with values in an lcHs $E$ as

$$
\mathcal{C V}^{k}(\Omega, E):=\left\{\left.f \in \mathcal{C}^{k}(\Omega, E)|\forall j \in J, l \in\langle k\rangle, \alpha \in \mathfrak{A}:| f\right|_{j, l, \alpha}<\infty\right\}
$$

where

$$
|f|_{j, l, \alpha}:=\sup _{\substack{x \in \Omega \\ \beta \in \mathbb{N}_{0}^{d},|\beta| \leq l}} p_{\alpha}\left(\left(\partial^{\beta}\right)^{E} f(x)\right) \nu_{j, l}(x) .
$$

We define the topological subspace of $\mathcal{C} \mathcal{V}^{k}(\Omega, E)$ consisting of the functions that vanish with all their derivatives when weighted at infinity by

$$
\begin{aligned}
\mathcal{C V}_{0}^{k}(\Omega, E):=\left\{f \in \mathcal{C V}^{k}(\Omega, E) \mid\right. & \forall j \in J, l \in\langle k\rangle, \alpha \in \mathfrak{A}, \varepsilon>0 \\
& \left.\exists K \subset \Omega \text { compact }:|f|_{\Omega \backslash K, j, l, \alpha}<\varepsilon\right\}
\end{aligned}
$$

where

$$
|f|_{\Omega \backslash K, j, l, \alpha}:=\sup _{\substack{x \in \Omega \backslash K \\ \beta \in \mathbb{N}_{0}^{d},|\beta| \leq l}} p_{\alpha}\left(\left(\partial^{\beta}\right)^{E} f(x)\right) \nu_{j, l}(x) .
$$

It is easily seen that these spaces are locally convex Hausdorff spaces with a directed system of seminorms due to our assumptions on the family $\mathcal{V}^{k}$ of weights.

REMARK 3.3. Suppose that in the definition of the space $\mathcal{C} V^{k}(\Omega, E)$ the weights also depend on $\beta \in \mathbb{N}_{0}^{d}$, i.e. the seminorms used to define $\mathcal{C} \mathcal{V}^{k}(\Omega, E)$ are of the form

$$
|f|_{j, l, \alpha}:=\sup _{\substack{x \in \Omega \\ \beta \in \mathbb{N}_{0}^{d},|\beta| \leq l}} p_{\alpha}\left(\left(\partial^{\beta}\right)^{E} f(x)\right) \nu_{j, l, \beta}(x) .
$$

Without loss of generality we may always use weights which are independent of $\beta$. Namely, by setting $\nu_{j, l}:=\max _{\beta \in \mathbb{N}_{0}^{d},|\beta| \leq l} \nu_{j, l, \beta}$ for $j \in J$ and $l \in\langle k\rangle$, we can switch to the usual system of seminorms $\left(|f|_{j, l, \alpha}\right)$ induced by the weights $\left(\nu_{j, l}\right)$ which is equivalent to $\left(|f|_{j, l, \alpha}\right)$.

The standard structure of a directed family $\mathcal{V}^{k}$ of weights on a locally compact Hausdorff space $\Omega$ is given by the following. Let $\left(\Omega_{j}\right)_{j \in J}$ be a family of sets such that $\Omega_{j} \subset \Omega_{j+1}$ 
for all $j \in J$ with $\Omega=\bigcup_{j \in J} \Omega_{j}$. Let $\widetilde{\nu}_{j, l}: \Omega \rightarrow(0, \infty)$ be continuous for all $j \in J$ and $l \in\langle k\rangle$, increasing in $j \in J$, i.e. $\widetilde{\nu}_{j, l} \leq \widetilde{\nu}_{j+1, l}$, and in $l \in\langle k\rangle$, i.e. $\widetilde{\nu}_{j, l} \leq \widetilde{\nu}_{j, l+1}$ if $l+1 \in\langle k\rangle$, such that

$$
\nu_{j, l}(x)=\chi_{\Omega_{j}}(x) \widetilde{\nu}_{j, l}(x), \quad x \in \Omega
$$

for every $j \in J$ and $l \in\langle k\rangle$ where $\chi_{\Omega_{j}}$ is the indicator function of $\Omega_{j}$. Further, we remark that the spaces $\mathcal{C} \mathcal{V}^{k}(\Omega, E)$ and $\mathcal{C} \mathcal{V}_{0}^{k}(\Omega, E)$ might coincide which is already mentioned in [2, 1.3 Bemerkung, p. 189] for $k=0$.

REMARK 3.4. If for every $j \in J$ and $l \in\langle k\rangle$ there are $i \in J$ and $m \in\langle k\rangle$ such that for all $\varepsilon>0$ there is a compact set $K \subset \Omega$ with $\nu_{j, l}(x) \leq \varepsilon \nu_{i, m}(x)$ for all $x \in \Omega \backslash K$, then $\mathcal{C V}^{k}(\Omega, E)=\mathcal{C V}_{0}^{k}(\Omega, E)$.

Examples of spaces where this happens are $\mathcal{C}^{k}(\Omega, E)$ with the topology of uniform convergence of all partial derivatives up to order $k$ on compact subsets of $\Omega$ and the Schwartz space $\mathcal{S}\left(\mathbb{R}^{d}, E\right)$.

EXAmple 3.5. Let $E$ be an lcHs, $k \in \mathbb{N}_{0, \infty}$ and $\Omega \subset \mathbb{R}^{d}$ open. Then

a) $\mathcal{C}^{k}(\Omega, E)=\mathcal{C W}^{k}(\Omega, E)=\mathcal{C W}_{0}^{k}(\Omega, E)$ with $\mathcal{W}^{k}:=\left\{\nu_{j, l}:=\chi_{\Omega_{j}} \mid j \in \mathbb{N}, l \in\langle k\rangle\right\}$ where $\left(\Omega_{j}\right)_{j \in \mathbb{N}}$ is a compact exhaustion of $\Omega$,

b) $\mathcal{S}\left(\mathbb{R}^{d}, E\right)=\mathcal{C} \mathcal{V}^{\infty}\left(\mathbb{R}^{d}, E\right)=\mathcal{C} \mathcal{V}_{0}^{\infty}\left(\mathbb{R}^{d}, E\right)$ with $\mathcal{V}^{\infty}:=\left\{\nu_{j, l} \mid j \in \mathbb{N}, l \in \mathbb{N}_{0}\right\}$ where $\nu_{j, l}(x):=\left(1+|x|^{2}\right)^{l / 2}$ for $x \in \mathbb{R}^{d}$.

Proof.

a) $\left(\Omega_{j}\right)_{j \in \mathbb{N}}$ being a compact exhaustion of $\Omega$ means that $\Omega=\bigcup_{j \in \mathbb{N}} \Omega_{j}, \Omega_{j}$ is compact and $\Omega_{j} \subset \stackrel{\Omega}{\Omega+1}_{j+1}$ for all $j \in \mathbb{N}$ where $\AA_{j+1}$ is the set of inner points of $\Omega_{j+1}$. For compact $\Omega_{j} \subset \Omega$ and $l \in\langle k\rangle$ our claim follows from Remark 3.4 with the choice $i:=j, m:=l$ and $K:=\Omega_{j}$.

b) We recall that the Schwartz space is defined by

$$
\mathcal{S}\left(\mathbb{R}^{d}, E\right):=\left\{f \in \mathcal{C}^{\infty}\left(\mathbb{R}^{d}, E\right) \mid \forall l \in \mathbb{N}_{0}, \alpha \in \mathfrak{A}:\|f\|_{l, \alpha}<\infty\right\}
$$

where

$$
\|f\|_{l, \alpha}:=\sup _{\substack{x \in \mathbb{R}^{d} \\ \beta \in \mathbb{N}_{0}^{d},|\beta| \leq l}} p_{\alpha}\left(\left(\partial^{\beta}\right)^{E} f(x)\right)\left(1+|x|^{2}\right)^{l / 2} .
$$

Thus $\mathcal{S}\left(\mathbb{R}^{d}, E\right)=\mathcal{C} \mathcal{V}^{\infty}\left(\mathbb{R}^{d}, E\right)$. We note that for every $j \in \mathbb{N}, l \in \mathbb{N}_{0}$ and $\varepsilon>0$ there is $r>0$ such that

$$
\frac{\nu_{j, l}(x)}{\nu_{j, 2(l+1)}(x)}=\frac{\left(1+|x|^{2}\right)^{l / 2}}{\left(1+|x|^{2}\right)^{l+1}}=\left(1+|x|^{2}\right)^{-(l / 2)-1}<\varepsilon
$$

for all $x \notin \overline{\mathbb{B}_{r}(0)}=: K$ yielding $\mathcal{S}\left(\mathbb{R}^{d}, E\right)=\mathcal{C} \mathcal{V}_{0}^{\infty}\left(\mathbb{R}^{d}, E\right)$ by Remark 3.4

The question of finite dimensional approximation from the introduction is closely connected to the property of a family of weights being locally bounded away from zero.

DEFinition 3.6 (locally bounded away from zero). Let $\Omega$ be a locally compact Hausdorff space and $k \in \mathbb{N}_{0, \infty}$. A family of weights $\mathcal{V}^{k}$ is called locally bounded away from zero on $\Omega$ if

$$
\forall K \subset \Omega \text { compact, } l \in\langle k\rangle \exists j \in J: \inf _{x \in K} \nu_{j, l}(x)>0 \text {. }
$$


For $k=0$ (and locally compact Hausdorff $\Omega$ ) this coincides with condition (ii) of Theorem 1.1. It even guarantees that the spaces $\mathcal{C} \mathcal{V}^{k}(\Omega, E)$ and $\mathcal{C} \mathcal{V}_{0}^{k}(\Omega, E)$ are complete for complete $E$.

Proposition 3.7. Let $E$ be a complete lcHs, $k \in \mathbb{N}_{0, \infty}$ and $\mathcal{V}^{k}$ be a family of weights which is locally bounded away from zero on a locally compact Hausdorff space $\Omega(k=0)$ or an open set $\Omega \subset \mathbb{R}^{d}(k>0)$. Then $\mathcal{C V}^{k}(\Omega, E)$ and $\mathcal{C} V_{0}^{k}(\Omega, E)$ are complete locally convex Hausdorff spaces. In particular, they are Fréchet spaces if $E$ is a Fréchet space and $J$ countable.

Proof. Let $\left(f_{\tau}\right)_{\tau \in \mathcal{T}}$ be a Cauchy net in $\mathcal{C V}^{k}(\Omega, E)$. The space $\mathcal{C}^{k}(\Omega, E)$ equipped with the usual system of seminorms $\left(q_{K, l, \alpha}\right)$ given in $(1)$ is complete by [23, Proposition 44.1, p. 446]. Let $K \subset \Omega$ compact, $l \in\langle k\rangle$ and $\alpha \in \mathfrak{A}$. Since $\mathcal{V}^{k}$ is locally bounded away from zero, there is $j \in J$ such that

$$
q_{K, l, \alpha}(f) \leq \sup _{x \in K} \nu_{j, l}(x)^{-1}|f|_{j, l, \alpha}=\left(\inf _{x \in K} \nu_{j, l}(x)\right)^{-1}|f|_{j, l, \alpha}, \quad f \in \mathcal{C V}^{k}(\Omega, E),
$$

implying that the inclusion $\mathcal{C V}^{k}(\Omega, E) \hookrightarrow \mathcal{C}^{k}(\Omega, E)$ is continuous. Thus $\left(f_{\tau}\right)$ is a Cauchy net in $\mathcal{C}^{k}(\Omega, E)$ as well and has a limit $f$ in this space due to the completeness. Let $j \in J$, $l \in\langle k\rangle, \alpha \in \mathfrak{A}$ and $\varepsilon>0$. As this convergence implies pointwise convergence, we have that for all $x \in \Omega$ and $\beta \in \mathbb{N}_{0}^{d},|\beta| \leq l$, there exists $\tau_{j, l, \beta, x} \in \mathcal{T}$ such that for all $\tau \geq \tau_{j, l, \beta, x}$

$$
p_{\alpha}\left(\left(\partial^{\beta}\right)^{E} f_{\tau}(x)-\left(\partial^{\beta}\right)^{E} f(x)\right)<\frac{\varepsilon}{2 \nu_{j, l}(x)}
$$

if $\nu_{j, l}(x)>0$. Furthermore, there exists $\tau_{0} \in \mathcal{T}$ such that for all $\tau, \mu \geq \tau_{0}$

$$
\left|f_{\tau}-f_{\mu}\right|_{j, l, \alpha}<\frac{\varepsilon}{2}
$$

by assumption. Hence we get for all $\tau \geq \tau_{0}$ by choosing $\mu \geq \tau_{j, l, \beta, x}, \tau_{0}$

$$
\begin{aligned}
& p_{\alpha}\left(\left(\partial^{\beta}\right)^{E} f(x)\right) \nu_{j, l}(x)-p_{\alpha}\left(\left(\partial^{\beta}\right)^{E} f_{\tau}(x)\right) \nu_{j, l}(x) \\
\leq & p_{\alpha}\left(\left(\partial^{\beta}\right)^{E} f_{\tau}(x)-\left(\partial^{\beta}\right)^{E} f(x)\right) \nu_{j, l}(x) \\
\leq & p_{\alpha}\left(\left(\partial^{\beta}\right)^{E} f_{\tau}(x)-\left(\partial^{\beta}\right)^{E} f_{\mu}(x)\right) \nu_{j, l}(x)+p_{\alpha}\left(\left(\partial^{\beta}\right)^{E} f_{\mu}(x)-\left(\partial^{\beta}\right)^{E} f(x)\right) \nu_{j, l}(x) \\
\leq & \sup _{z \in \Omega} p_{\alpha}\left(\left(\partial^{\beta}\right)^{E} f_{\tau}(z)-\left(\partial^{\beta}\right)^{E} f_{\mu}(z)\right) \nu_{j, l}(z)+\frac{\varepsilon}{2} \\
\leq & \sup _{\substack{z \in \Omega \\
\gamma \in \mathbb{N}_{0}^{d},|\gamma| \leq l}} p_{\alpha}\left(\left(\partial^{\gamma}\right)^{E} f_{\tau}(z)-\left(\partial^{\gamma}\right)^{E} f_{\mu}(z)\right) \nu_{j, l}(z)+\frac{\varepsilon}{2}=\left|f_{\tau}-f_{\mu}\right|_{j, l, \alpha}+\frac{\varepsilon}{2} \varepsilon \\
& \text { 3. }
\end{aligned}
$$

if $\nu_{j, l}(x)>0$. We deduce that for all $\tau \geq \tau_{0}$

$$
\begin{aligned}
& p_{\alpha}\left(\left(\partial^{\beta}\right)^{E} f(x)\right) \nu_{j, l}(x)-p_{\alpha}\left(\left(\partial^{\beta}\right)^{E} f_{\tau}(x)\right) \nu_{j, l}(x) \\
\leq & p_{\alpha}\left(\left(\partial^{\beta}\right)^{E} f_{\tau}(x)-\left(\partial^{\beta}\right)^{E} f(x)\right) \nu_{j, l}(x)<\varepsilon
\end{aligned}
$$

if $\nu_{j, l}(x)>0$. If $\nu_{j, l}(x)=0$, then this estimate is also fulfilled and so $\left|f_{\tau}-f\right|_{j, l, \alpha} \leq \varepsilon$ as well as $|f|_{j, l, \alpha} \leq \varepsilon+\left|f_{\tau}\right|_{j, l, \alpha}$ for all $\tau \geq \tau_{0}$. This means that $f \in \mathcal{C V}^{k}(\Omega, E)$ and that $\left(f_{\tau}\right)$ converges to $f$ in $\mathcal{C} \mathcal{V}^{k}(\Omega, E)$. Therefore $\mathcal{C} \mathcal{V}^{k}(\Omega, E)$ is complete and $\mathcal{C} \mathcal{V}_{0}^{k}(\Omega, E)$ as well because it is a closed subspace of the complete space $\mathcal{C} \mathcal{V}^{k}(\Omega, E)$. 
For $k \in \mathbb{N}_{0, \infty}$ and locally compact Hausdorff $\Omega(k=0)$ or open $\Omega \subset \mathbb{R}^{d}(k>0)$ we define $\mathcal{C} \mathcal{V}_{c}^{k}(\Omega, E)$ to be the subspace of $\mathcal{C V}^{k}(\Omega, E)$ of functions with compact support. Obviously we have $\mathcal{C} \mathcal{V}_{c}^{k}(\Omega, E) \subset \mathcal{C} \mathcal{V}_{0}^{k}(\Omega, E)$ and $\mathcal{C} \mathcal{V}_{c}^{k}(\Omega, E) \subset \mathcal{C}_{c}^{k}(\Omega, E)$. On the other hand, the space $\mathcal{C}_{c}^{k}(\Omega, E)$ is a linear subspace of $\mathcal{C} \mathcal{V}_{c}^{k}(\Omega, E)$ if the family of weights $\mathcal{V}^{k}$ fulfils the definition of local boundedness.

DEFinition 3.8 (locally bounded). Let $\Omega$ be a locally compact Hausdorff space and $k \in \mathbb{N}_{0, \infty}$. A family of weights $\mathcal{V}^{k}$ is called locally bounded on $\Omega$ if

$$
\forall K \subset \Omega \text { compact, } j \in J, l \in\langle k\rangle: \sup _{x \in K} \nu_{j, l}(x)<\infty .
$$

Indeed, if $f \in \mathcal{C}_{c}^{k}(\Omega, E)$, then we have for $K:=\operatorname{supp} f$

$$
|f|_{j, l, \alpha}=\sup _{\substack{x \in K \\ \beta \in \mathbb{N}_{0}^{d},|\beta| \leq l}} p_{\alpha}\left(\left(\partial^{\beta}\right)^{E} f(x)\right) \nu_{j, l}(x) \leq\left(\sup _{\substack{z \in K \\ \beta \in \mathbb{N}_{0}^{d},|\beta| \leq l}} p_{\alpha}\left(\left(\partial^{\beta}\right)^{E} f(z)\right)\right) \sup _{x \in K} \nu_{j, l}(x)
$$

for all $j \in J, l \in\langle k\rangle$ and $\alpha \in \mathfrak{A}$. Hence we have:

REMARK 3.9. Let $E$ be an lcHs and $k \in \mathbb{N}_{0, \infty}$. If $\mathcal{V}^{k}$ is a family of locally bounded weights, then $\mathcal{C}_{c}^{k}(\Omega, E)=\mathcal{C} \mathcal{V}_{c}^{k}(\Omega, E)$ algebraically.

Next, we phrase a sufficient criterion for the density of $\mathcal{C}_{c}^{k}(\Omega, E)$ in $\mathcal{C V}_{0}^{k}(\Omega, E)$ for $k \in \mathbb{N}_{0, \infty}, \Omega \subset \mathbb{R}^{d}$ open and locally bounded $\mathcal{V}^{k}$.

Definition 3.10 (cut-off criterion). Let $E$ be an lcHs, $k \in \mathbb{N}_{0, \infty}, \Omega \subset \mathbb{R}^{d}$ open and $\mathcal{V}^{k}$ be a family of weights on $\Omega$. We say that $\mathcal{C} \mathcal{V}_{0}^{k}(\Omega, E)$ satisfies the cut-off criterion if

$$
\forall f \in \mathcal{C V}_{0}^{k}(\Omega, E), j \in J, l \in\langle k\rangle, \alpha \in \mathfrak{A} \exists \delta>0 \forall \varepsilon>0 \exists K \subset \Omega \text { compact : }
$$

$$
\left(K+\overline{\mathbb{B}_{\delta}(0)}\right) \subset \Omega \text { and }|f|_{\Omega \backslash K, j, l, \alpha}<\varepsilon .
$$

REMARK 3.11. If $\Omega=\mathbb{R}^{d}$, then the cut-off criterion is satisfied for any $\delta>0$.

EXAmPLE 3.12. Let $E$ be an lcHs, $k \in \mathbb{N}_{0, \infty}$ and $\Omega \subset \mathbb{R}^{d}$ open. The space $\mathcal{C}^{k}(\Omega, E)$ with the usual topology of uniform convergence of all partial derivatives up to order $k$ on compact subsets of $\Omega$ and the Schwartz space $\mathcal{S}\left(\mathbb{R}^{d}, E\right)$ fulfil the cut-off criterion.

Proof. For the Schwartz space this follows directly from Example $3.5 \mathrm{~b}$ ) and Remark 3.11 By Example 3.5 a) we have $\mathcal{C}^{k}(\Omega, E)=\mathcal{C} \mathcal{W}_{0}^{k}(\Omega, E)$ with $\mathcal{W}^{k}:=\left\{\nu_{j, l}:=\chi_{\Omega_{j}} \mid j \in \mathbb{N}\right.$, $l \in\langle k\rangle\}$ where $\left(\Omega_{j}\right)_{j \in \mathbb{N}}$ is a compact exhaustion of $\Omega$. Choosing $K:=\Omega_{j}$ and $\delta:=$ $\inf \left\{|z-x| \mid z \in \partial \Omega_{j}, x \in \partial \Omega_{j+1}\right\}>0$ for $j \in \mathbb{N}$, we note that the cut-off criterion is fulfilled.

The proof of the density given below uses cut-off functions and the additional $\delta>0$ independent of $\varepsilon>0$ allows us to choose a suitable cut-off function whose derivatives can be estimated independently of $\varepsilon$. But first we recall the following definitions since we need the product rule. Let $\gamma, \beta \in \mathbb{N}_{0}^{d}$. We write $\gamma \leq \beta$ if $\gamma_{n} \leq \beta_{n}$ for all $1 \leq n \leq d$, and define

$$
\left(\begin{array}{l}
\beta \\
\gamma
\end{array}\right):=\prod_{n=1}^{d}\left(\begin{array}{l}
\beta_{n} \\
\gamma_{n}
\end{array}\right)
$$


if $\gamma \leq \beta$ where the right-hand side is defined by ordinary binomial coefficients. Now, we can phrase the product rule whose proof follows by induction (just adapt the proof for scalar-valued functions).

Proposition 3.13 (product rule). Let $E$ be an lcHs, $k \in \mathbb{N}_{0, \infty}, \Omega \subset \mathbb{R}^{d}$ open, $f \in$ $\mathcal{C}^{k}(\Omega, E)$ and $g \in \mathcal{C}^{k}(\Omega)$. Then $g f \in \mathcal{C}^{k}(\Omega, E)$ and

$$
\left(\partial^{\beta}\right)^{E}(g f)(x)=\sum_{\gamma \leq \beta}\left(\begin{array}{l}
\beta \\
\gamma
\end{array}\right)\left(\partial^{\beta-\gamma}\right)^{\mathbb{K}} g(x)\left(\partial^{\gamma}\right)^{E} f(x), \quad x \in \Omega, \beta \in \mathbb{N}_{0}^{d},|\beta| \leq k .
$$

LEMMA 3.14. Let $E$ be an lcHs, $k \in \mathbb{N}_{0, \infty}$ and $\mathcal{V}^{k}$ be a family of locally bounded weights on an open set $\Omega \subset \mathbb{R}^{d}$. If $\mathcal{C} \mathcal{V}_{0}^{k}(\Omega, E)$ satisfies the cut-off criterion, then the space $\mathcal{C}_{c}^{k}(\Omega, E)$ is dense in $\mathcal{C V}_{0}^{k}(\Omega, E)$.

Proof. The local boundedness of $\mathcal{V}^{k}$ yields that $\mathcal{C}_{c}^{k}(\Omega, E)$ is a linear subspace of $\mathcal{C} \mathcal{V}_{0}^{k}(\Omega, E)$ by Remark 3.9 which we equip with the induced topology. Let $f \in \mathcal{C V}_{0}^{k}(\Omega, E), j \in J$, $l \in\langle k\rangle$ and $\alpha \in \mathfrak{A}$. Due to the cut-off criterion there is $\delta>0$ such that for $\varepsilon>0$ there is $K \subset \Omega$ compact with $\left(K+\overline{\mathbb{B}_{\delta}(0)}\right) \subset \Omega$ and $|f|_{\Omega \backslash K, j, l, \alpha}<\varepsilon$. We choose a cut-off function $\psi \in \mathcal{C}_{c}^{\infty}(\Omega)$ with $0 \leq \psi \leq 1$ so that $\psi=1$ in a neighbourhood of $K$ and

$$
\left|\left(\partial^{\beta}\right)^{\mathbb{K}} \psi\right| \leq C_{\beta} \delta^{-|\beta|}
$$

on $\Omega$ for all $\beta \in \mathbb{N}_{0}^{d}$ where $C_{\beta}>0$ only depends on $\beta$ (see [12, Theorem 1.4.1, p. 25]). We set $K_{0}:=\operatorname{supp} \psi$, note that $\psi f \in \mathcal{C}_{c}^{k}(\Omega, E)$ by the product rule and

$$
\begin{aligned}
& |f-\psi f|_{j, l, \alpha}=\sup _{\substack{x \in \Omega \backslash K \\
\beta \in \mathbb{N}_{0}^{d},|\beta| \leq l}} p_{\alpha}\left(\left(\partial^{\beta}\right)^{E}(f-\psi f)(x)\right) \nu_{j, l}(x) \\
& \leq \sup _{\substack{x \in \Omega \backslash K \\
\beta \in \mathbb{N}_{0}^{d},|\beta| \leq l}} p_{\alpha}\left(\left(\partial^{\beta}\right)^{E} f(x)\right) \nu_{j, l}(x)+\sup _{\substack{x \in \Omega \backslash K \\
\beta \in \mathbb{N}_{0}^{d},|\beta| \leq l}} p_{\alpha}\left(\left(\partial^{\beta}\right)^{E}(\psi f)(x)\right) \nu_{j, l}(x) \\
& =|f|_{\Omega \backslash K, j, l, \alpha}+\sup _{\substack{x \in(\Omega \backslash K) \cap K_{0} \\
\beta \in \mathbb{N}_{0}^{d},|\beta| \leq l}} p_{\alpha}\left(\sum_{\gamma \leq \beta}\left(\begin{array}{l}
\beta \\
\gamma
\end{array}\right)\left(\partial^{\beta-\gamma}\right)^{\mathbb{K}} \psi(x)\left(\partial^{\gamma}\right)^{E} f(x)\right) \nu_{j, l}(x) \\
& \leq|f|_{\Omega \backslash K, j, l, \alpha}+\sup _{\substack{z \in K_{0} \\
\beta \in \mathbb{N}_{0}^{d},|\beta| \leq l}} \sum_{\gamma \leq \beta}\left(\begin{array}{l}
\beta \\
\gamma
\end{array}\right)\left|\left(\partial^{\beta-\gamma}\right)^{\mathbb{K}} \psi(z)\right|\left(\sup _{\substack{x \in \Omega \backslash K \\
\tau \in \mathbb{N}_{0}^{d},|\tau| \leq l}} p_{\alpha}\left(\left(\partial^{\tau}\right)^{E} f(x)\right) \nu_{j, l}(x)\right) \\
& \leq|f|_{\Omega \backslash K, j, l, \alpha}+\underbrace{\sup _{\beta \in \mathbb{N}_{0}^{d},|\beta| \leq l} \sum_{\gamma \leq \beta}\left(\begin{array}{l}
\beta \\
\gamma
\end{array}\right) C_{\beta-\gamma} \delta^{-|\beta-\gamma|}}_{=: C_{l, \delta}<\infty}|f|_{\Omega \backslash K, j, l, \alpha} \\
& =\left(1+C_{l, \delta}\right)|f|_{\Omega \backslash K, j, l, \alpha}<\left(1+C_{l, \delta}\right) \varepsilon .
\end{aligned}
$$

The independence of $C_{l, \delta}$ from $\varepsilon$ implies the statement.

We complete this section by pointing out the link between our question on finite dimensional approximation and the tensor product. If $\mathcal{V}^{k}$ is locally bounded away from zero, there is a nice relation between our spaces of vector-valued functions and the $\varepsilon$-product which uses that the point-evaluation functionals $\delta_{x}: f \mapsto f(x)$ are continuous on $\mathcal{C V}^{k}(\Omega)$ by our definition of a weight. 
Proposition 3.15. Let $E$ be an $l c H s, k \in \mathbb{N}_{0, \infty}, \mathcal{V}^{k}$ be a family of weights which is locally bounded away from zero on a locally compact Hausdorff space $\Omega(k=0)$ or an open set $\Omega \subset \mathbb{R}^{d}(k>0)$.

a) In addition, let $\mathcal{C V}_{0}^{k}(\Omega)$ be barrelled if $k>0$. Then

$$
S_{\mathcal{C} \mathcal{V}_{0}^{k}(\Omega)}: \mathcal{C V}_{0}^{k}(\Omega) \varepsilon E \rightarrow \mathcal{C V}_{0}^{k}(\Omega, E), u \longmapsto\left[x \mapsto u\left(\delta_{x}\right)\right],
$$

is an isomorphism into, i.e. an isomorphism to its range.

b) In addition, let $\mathcal{C V}^{k}(\Omega)$ be barrelled if $k>0$. Then

$$
S_{\mathcal{C V}^{k}(\Omega)}: \mathcal{C V}^{k}(\Omega) \varepsilon E \rightarrow \mathcal{C V}^{k}(\Omega, E), u \longmapsto\left[x \mapsto u\left(\delta_{x}\right)\right]
$$

is an isomorphism into.

Proof. Let $u \in \mathcal{C V}_{0}^{k}(\Omega) \varepsilon E$, resp. $\mathcal{C} V^{k}(\Omega) \varepsilon E$, and as a simplification we omit the index of $S$. The continuity of $S(u)$ is a consequence of [17, 4.1 Proposition, p. 18] and [17, 4.2 Lemma (i), p. 19] since $\mathcal{V}^{k}$ is locally bounded away from zero. If $k>0$, then the continuous partial differentiability of $S(u)$ up to order $k$ follows from [17, 4.12 Proposition, p. 22] as $\mathcal{C} \mathcal{V}_{0}^{k}(\Omega)$, resp. $\mathcal{C} \mathcal{V}^{k}(\Omega)$, is barrelled and $\mathcal{V}^{k}$ locally bounded away from zero. If $u \in \mathcal{C} \mathcal{V}_{0}^{k}(\Omega) \varepsilon E$, then $S(u)$ vanishes together with all its derivatives when weighted at infinity by [17, 4.13 Proposition, p. 23]. Thanks to these observations [17, 3.9 Theorem, p. 9] proves our statement.

In particular, if $J$ is countable and $\mathcal{V}^{k}$ locally bounded away from zero, then the Fréchet spaces $\mathcal{C} \mathcal{V}^{k}(\Omega)$ and $\mathcal{C} V_{0}^{k}(\Omega)$ are barrelled. This result allows us to identify the injective tensor product of $\mathcal{C} \mathcal{V}^{k}(\Omega)$, resp. $\mathcal{C} \mathcal{V}_{0}^{k}(\Omega)$, and $E$ with a subspace of $\mathcal{C} \mathcal{V}^{k}(\Omega, E)$, resp. $\mathcal{C} \mathcal{V}_{0}^{k}(\Omega, E)$. Let us use the symbol $\mathcal{F}$ for $\mathcal{C} \mathcal{V}^{k}$ or $\mathcal{C} \mathcal{V}_{0}^{k}$. We consider $\mathcal{F}(\Omega) \otimes E$ as an algebraic subspace of $\mathcal{F}(\Omega) \varepsilon E$ by means of the linear injection

$$
\Theta_{\mathcal{F}(\Omega)}: \mathcal{F}(\Omega) \otimes E \rightarrow \mathcal{F}(\Omega) \varepsilon E, \quad \sum_{n=1}^{m} f_{n} \otimes e_{n} \longmapsto\left[y \mapsto \sum_{n=1}^{m} y\left(f_{n}\right) e_{n}\right] .
$$

Via $\Theta_{\mathcal{F}(\Omega)}$ the topology of $\mathcal{F}(\Omega) \varepsilon E$ induces a locally convex topology on $\mathcal{F}(\Omega) \otimes E$ and $\mathcal{F}(\Omega) \otimes_{\varepsilon} E$ denotes $\mathcal{F}(\Omega) \otimes E$ equipped with this topology. From the preceding proposition and the composition $S_{\mathcal{F}(\Omega)} \circ \Theta_{\mathcal{F}(\Omega)}$ we obtain:

COROLlary 3.16. Let $E$ be an lcHs, $k \in \mathbb{N}_{0, \infty}, \mathcal{V}^{k}$ be a family of weights which is locally bounded away from zero on a locally compact Hausdorff space $\Omega(k=0)$ or an open set $\Omega \subset \mathbb{R}^{d}(k>0)$. Fix the notation $\mathcal{F}=\mathcal{C V}^{k}$ or $\mathcal{C} \mathcal{V}_{0}^{k}$ and let $\mathcal{F}(\Omega)$ be barrelled if $k>0$.

a) We get by identification of isomorphic subspaces

$$
\mathcal{F}(\Omega) \otimes_{\varepsilon} E \subset \mathcal{F}(\Omega) \varepsilon E \subset \mathcal{F}(\Omega, E)
$$

and the embedding $\mathcal{F}(\Omega) \otimes E \hookrightarrow \mathcal{F}(\Omega, E)$ is given by $f \otimes e \mapsto[x \mapsto f(x) e]$.

b) Let $\mathcal{F}(\Omega)$ and $E$ be complete. If $\mathcal{F}(\Omega) \otimes E$ is dense in $\mathcal{F}(\Omega, E)$, then

$$
\mathcal{F}(\Omega, E) \cong \mathcal{F}(\Omega) \varepsilon E \cong \mathcal{F}(\Omega) \widehat{\otimes}_{\varepsilon} E .
$$

In particular, $\mathcal{F}(\Omega)$ has the approximation property if $\mathcal{F}(\Omega) \otimes E$ is dense in $\mathcal{F}(\Omega, E)$ for every complete $E$. 
Proof.

a) The inclusions hold by Proposition 3.15 and $\mathcal{F}(\Omega) \varepsilon E$ and $\mathcal{F}(\Omega, E)$ induce the same topology on $\mathcal{F}(\Omega) \otimes E$. Further, we have

$$
f \otimes e \stackrel{\Theta_{\mathcal{F}(\Omega)}}{\longmapsto}[y \mapsto y(f) e] \stackrel{S_{\mathcal{F}(\Omega)}}{\longmapsto}\left[x \longmapsto[y \mapsto y(f) e]\left(\delta_{x}\right)\right]=[x \mapsto f(x) e] .
$$

b) If $\mathcal{F}(\Omega)$ and $E$ are complete, then we obtain that $\mathcal{F}(\Omega) \varepsilon E$ is complete by [15, Satz 10.3 , p. 234]. In addition, we get the completion of $\mathcal{F}(\Omega) \otimes_{\varepsilon} E$ as its closure in $\mathcal{F}(\Omega) \varepsilon E$ which coincides with the closure in $\mathcal{F}(\Omega, E)$. The rest follows directly from a).

Looking at part a), we derive

$$
\left(S_{\mathcal{F}(\Omega)} \circ \Theta_{\mathcal{F}(\Omega)}\right)\left(\sum_{n=1}^{m} f_{n} \otimes e_{n}\right)=\sum_{n=1}^{m} f_{n} e_{n}
$$

for $m \in \mathbb{N}, f_{n} \in \mathcal{F}(\Omega)$ and $e_{n} \in E, 1 \leq n \leq m$. Hence we see that the answer to our question is affirmative if $\mathcal{F}(\Omega) \otimes E$ is dense in $\mathcal{F}(\Omega, E)$. For the sake of completeness we remark the following.

Proposition 3.17. Let $E$ be an lcHs, $k \in \mathbb{N}_{0, \infty}, \mathcal{V}^{k}$ be a family of weights which is locally bounded away from zero on a locally compact Hausdorff space $\Omega(k=0)$ or an open set $\Omega \subset \mathbb{R}^{d}(k>0)$.

a) In addition, let $\mathcal{C} \mathcal{V}_{0}^{k}(\Omega)$ be barrelled if $k>0$. If $E$ is quasi-complete and $\mathcal{V}^{k}$ locally bounded on $\Omega$, then

$$
\mathcal{C} \mathcal{V}_{0}^{k}(\Omega) \varepsilon E \cong \mathcal{C} \mathcal{V}_{0}^{k}(\Omega, E) \quad \text { via } S_{\mathcal{C} \mathcal{V}_{0}^{k}(\Omega)}
$$

b) In addition, let $\mathcal{C} \mathcal{V}^{k}(\Omega)$ be barrelled if $k>0$. If $E$ is a semi-Montel space, then

$$
\mathcal{C V}^{k}(\Omega) \varepsilon E \cong \mathcal{C V}^{k}(\Omega, E) \quad \text { via } S_{\mathcal{C} \mathcal{V}^{k}(\Omega)} \text {. }
$$

Proof. For $k>0$ this is [17, 5.10 Example a), p. 28], resp. [17, 3.21 Example a), p. 14]. Statement a) for $k=0$ is a consequence of [17, 3.20 Corollary, p. 13] in combination with [17, 4.1 Proposition, p. 18], [17, 4.2 Lemma (i), p. 19] and [17, 4.13 Proposition, p. 23]. For $k=0$ statement b) follows from [17, 3.19 Corollary, p. 13] in combination with [17. 4.1 Proposition, p. 18] and [17, 4.2 Lemma (i), p. 19].

The corresponding results for $k=0$ and a Nachbin-family $\mathcal{V}^{0}$ of weights are given in [3, 2.4 Theorem, p. 138-139] and [3, 2.12 Satz, p. 141]. In combination with our preceding observation, we deduce that every element of $\mathcal{C} \mathcal{V}_{0}^{k}(\Omega, E)$ can be approximated in $\mathcal{C V}_{0}^{k}(\Omega, E)$ by functions with values in a finite dimensional subspace if $E$ is a quasicomplete space with approximation property and the assumptions of the proposition above are fulfilled. The same is true for $\mathcal{C V}^{k}(\Omega, E)$ if $E$ is a semi-Montel space with approximation property. Due to the strong conditions on $E$ this is not really satisfying but actually the best we get for general $\mathcal{C} \mathcal{V}^{k}(\Omega, E)$. For $\mathcal{C} \mathcal{V}_{0}^{k}(\Omega, E)$ there is a better result available, whose proof we prepare on the next pages.

4. Convolution via the Pettis-integral. In this section we review the notion of the Pettis-integral. Trèves uses the Riemann-integral to define the convolution $f * g$ of a function $f \in \mathcal{C}_{c}^{k}(\Omega, E)$ and a function $g \in \mathcal{C}_{c}^{\infty}\left(\mathbb{R}^{d}\right)$ in the proof of Theorem 1.2 and states 
(without a proof) that the convolution defined in this way is a function in $\mathcal{C}_{c}^{\infty}\left(\mathbb{R}^{d}, \widehat{E}\right)$ and has all the properties known from the convolution of two scalar-valued functions. We use the Pettis-integral instead to define the convolution. The reason is that we can use the dominated convergence theorem for the Pettis-integral [19, Theorem 2, p. 162-163] to get the Leibniz' rule for differentiation under the integral sign which enables us to prove that the convolution has some of the key properties known from the scalar-valued case.

Let us fix some notation first. For a measure space $(X, \Sigma, \mu)$ let

$$
\mathfrak{L}^{1}(X, \mu):=\left\{f: X \rightarrow \mathbb{K} \text { measurable }\left|q_{1}(f):=\int_{X}\right| f(x) \mid \mathrm{d} \mu(x)<\infty\right\}
$$

and define the quotient space of integrable functions with respect to the measure $\mu$ by $\mathcal{L}^{1}(X, \mu):=\mathfrak{L}^{1}(X, \mu) /\left\{f \in \mathfrak{L}^{1}(X, \mu) \mid q_{1}(f)=0\right\}$. From now on we do not distinguish between equivalence classes and their representatives anymore. We say that $f: X \rightarrow \mathbb{K}$ is integrable on $\Lambda \in \Sigma$ and write $f \in \mathcal{L}^{1}(\Lambda, \mu)$ if $\chi_{\Lambda} f \in \mathcal{L}^{1}(X, \mu)$ where $\chi_{\Lambda}$ is the characteristic function of $\Lambda$. Then we set

$$
\int_{\Lambda} f(x) \mathrm{d} \mu(x):=\int_{X} \chi_{\Lambda}(x) f(x) \mathrm{d} \mu(x) .
$$

Definition 4.1 (Pettis-integral). Let $(X, \Sigma, \mu)$ be a measure space and $E$ an lcHs. A function $f: X \rightarrow E$ is called weakly (scalarly) measurable if the function $e^{\prime} \circ f: X \rightarrow \mathbb{K}$, $\left(e^{\prime} \circ f\right)(x):=\left\langle e^{\prime}, f(x)\right\rangle:=e^{\prime}(f(x))$, is measurable for all $e^{\prime} \in E^{\prime}$. A weakly measurable function is said to be weakly (scalarly) integrable if $e^{\prime} \circ f \in \mathcal{L}^{1}(X, \mu)$. A function $f: X \rightarrow E$ is called Pettis-integrable on $\Lambda \in \Sigma$ if it is weakly integrable on $\Lambda$ and

$$
\exists e_{\Lambda} \in E \forall e^{\prime} \in E^{\prime}:\left\langle e^{\prime}, e_{\Lambda}\right\rangle=\int_{\Lambda}\left\langle e^{\prime}, f(x)\right\rangle \mathrm{d} \mu(x) .
$$

In this case $e_{\Lambda}$ is unique due to $E$ being Hausdorff and we set

$$
\int_{\Lambda} f(x) \mathrm{d} \mu(x):=e_{\Lambda}
$$

A function $f$ is called Pettis-integrable on $\Sigma$ if it is Pettis-integrable on all $\Lambda \in \Sigma$.

We write $\mathcal{N}_{\mu}$ for the set of $\mu$-null sets of a measure space $(X, \Sigma, \mu)$ and for $\Lambda \in \Sigma$ we use the notion $\left(\Lambda, \Sigma_{\mid \Lambda}, \mu_{\mid \Lambda}\right)$ for the restricted measure space given by $\Sigma_{\mid \Lambda}:=\{\omega \in \Sigma \mid$ $\omega \subset \Lambda\}$ and $\mu_{\mid \Lambda}:=\mu_{\mid \Sigma_{\mid \Lambda}}$. If we consider the measure space $\left(\mathbb{R}^{d}, \mathscr{L}\left(\mathbb{R}^{d}\right), \lambda\right)$ of Lebesgue measurable sets, we just write $\mathrm{d} x:=\mathrm{d} \lambda(x)$.

REMARK 4.2. Let $(X, \Sigma, \mu)$ be a measure space, $E$ an lcHs and $f$ Pettis-integrable on $\Lambda \in \Sigma$. If $\omega \in \Sigma$ such that $\omega \subset \Lambda$ and $(\Lambda \backslash \omega) \subset\{x \in X \mid f(x)=0\}$, then $f$ is Pettis-integrable on $\omega$ and

$$
\int_{\omega} f(x) \mathrm{d} \mu(x)=\int_{\Lambda} f(x) \mathrm{d} \mu(x) .
$$

This follows directly from

$$
\left\langle e^{\prime}, \int_{\Lambda} f(x) \mathrm{d} \mu(x)\right\rangle=\int_{\Lambda}\left\langle e^{\prime}, f(x)\right\rangle \mathrm{d} \mu(x)=\int_{\omega}\left\langle e^{\prime}, f(x)\right\rangle \mathrm{d} \mu(x), \quad e^{\prime} \in E^{\prime} .
$$


Lemma 4.3. Let $E$ be a quasi-complete $l c H s,(X, \Sigma, \mu)$ a measure space, $T$ a metric space and suppose that $f: X \times T \rightarrow E$ fulfils the following conditions.

a) $f(\cdot, t)$ is Pettis-integrable on $\Sigma$ for all $t \in T$,

b) $f(x, \cdot): T \rightarrow E$ is continuous in a point $t_{0} \in T$ for $\mu$-almost all $x \in X$,

c) there is a neighbourhood $U \subset T$ of $t_{0}$ and a Pettis-integrable function $\psi$ on $\Sigma$ such that

$$
\forall t \in U, e^{\prime} \in E^{\prime} \exists N \in \mathcal{N}_{\mu} \forall x \in X \backslash N:\left|\left\langle e^{\prime}, f(x, t)\right\rangle\right| \leq\left|\left\langle e^{\prime}, \psi(x)\right\rangle\right| .
$$

Then $g_{\Lambda}: T \rightarrow E, g_{\Lambda}(t):=\int_{\Lambda} f(x, t) \mathrm{d} \mu(x)$, is well-defined and continuous in $t_{0}$ for every $\Lambda \in \Sigma$.

Proof. Let $\Lambda \in \Sigma$ and $\left(t_{n}\right)$ be a sequence in $U$ converging to $t_{0}$. From the continuous dependency of a scalar integral on a parameter (see [7, 5.6 Satz, p. 147]) we derive

$$
\lim _{n \rightarrow \infty} \int_{\Lambda}\langle e^{\prime}, \underbrace{f\left(x, t_{n}\right)}_{=: f_{n}(x)}\rangle \mathrm{d} \mu(x)=\int_{\Lambda}\langle e^{\prime}, \underbrace{f\left(x, t_{0}\right)}_{=: \widetilde{f}(x)}\rangle \mathrm{d} \mu(x) .
$$

For $n \in \mathbb{N}$ and $e^{\prime} \in E^{\prime}$ there is $N \in \mathcal{N}_{\mu}$ such that

$$
\left|\left\langle e^{\prime}, f_{n}(x)\right\rangle\right|=\left|\left\langle e^{\prime}, f\left(x, t_{n}\right)\right\rangle\right| \leq\left|\left\langle e^{\prime}, \psi(x)\right\rangle\right|
$$

for every $x \in X \backslash N$. Due to $(6)$ for every $\Lambda \in \Sigma$ and $e^{\prime} \in E^{\prime}$, (7) and the quasicompleteness of $E$ we can apply the dominated convergence theorem for the Pettisintegral [19, Theorem 2, p. 162-163] and deduce

$$
\lim _{n \rightarrow \infty} g_{\Lambda}\left(t_{n}\right)=\lim _{n \rightarrow \infty} \int_{\Lambda} f_{n}(x) \mathrm{d} \mu(x)=\int_{\Lambda} \widetilde{f}(x) \mathrm{d} \mu(x)=g_{\Lambda}\left(t_{0}\right) .
$$

The next lemma is the Leibniz' rule for differentiation under the integral sign for the Pettis-integral.

Lemma 4.4 (Leibniz' rule). Let $E$ be a quasi-complete lcHs, $(X, \Sigma, \mu)$ a measure space, $T \subset \mathbb{R}^{d}$ open and suppose that $f: X \times T \rightarrow E$ fulfils the following conditions.

a) $f(\cdot, t)$ is Pettis-integrable on $\Sigma$ for all $t \in T$,

b) there is a $\mu$-null set $N_{0} \in \mathcal{N}_{\mu}$ with $f(x, \cdot) \in \mathcal{C}^{1}(T, E)$ for all $x \in X \backslash N_{0}$,

c) for every $j \in \mathbb{N}, 1 \leq j \leq d$, there is a Pettis-integrable function $\psi_{j}$ on $\Sigma$ such that

$$
\forall e^{\prime} \in E^{\prime} \exists N \in \mathcal{N}_{\mu} \forall x \in X \backslash\left(N \cup N_{0}\right):\left|\left(\partial_{t_{j}}\right)^{\mathbb{K}}\left\langle e^{\prime}, f(x, \cdot)\right\rangle\right| \leq\left|\left\langle e^{\prime}, \psi_{j}(x)\right\rangle\right| .
$$

Then $g_{\Lambda}: T \rightarrow E, g_{\Lambda}(t):=\int_{\Lambda} f(x, t) \mathrm{d} \mu(x)$, is well-defined for every $\Lambda \in \Sigma, g_{\Lambda} \in$ $\mathcal{C}^{1}(T, E)$ and

$$
\left(\partial_{t_{j}}\right)^{E} g_{\Lambda}(t)=\int_{\Lambda}\left(\partial_{t_{j}}\right)^{E} f(x, t) \mathrm{d} \mu(x), \quad t \in T .
$$

Proof. First, we consider the case $\mathbb{K}=\mathbb{R}$. Let $\Lambda \in \Sigma, j \in \mathbb{N}, 1 \leq j \leq d, t \in T$ and $\left(h_{n}\right)$ be a real sequence converging to 0 such that $h_{n} \neq 0$ and $t+h_{n} e_{j} \in T$ for all $n$ where $e_{j}$ is the $j$-th unit vector in $\mathbb{R}^{d}$. Then

$$
\frac{g_{\Lambda}\left(t+h_{n} e_{j}\right)-g_{\Lambda}(t)}{h_{n}}=\int_{\Lambda} \underbrace{\frac{f\left(x, t+h_{n} e_{j}\right)-f(x, t)}{h_{n}}}_{=: f_{n}(x)} \mathrm{d} \mu(x) .
$$


We define the function $\tilde{f}: X \rightarrow E$ given by $\tilde{f}(x):=\left(\partial_{t_{j}}\right)^{E} f(x, t)$ for $x \in X \backslash N_{0}$ and $\widetilde{f}(x):=0$ for $x \in N_{0}$. We observe that

$$
\begin{aligned}
\lim _{n \rightarrow \infty} \int_{\Lambda}\left\langle e^{\prime}, f_{n}(x)\right\rangle \mathrm{d} \mu(x) & =\int_{\Lambda}\left(\partial_{t_{j}}\right)^{\mathbb{K}}\left\langle e^{\prime}, f(x, t)\right\rangle \mathrm{d} \mu(x) \\
& =\int_{\Lambda}\left\langle e^{\prime}, \partial_{t_{j}}^{E} f(x, t)\right\rangle \mathrm{d} \mu(x)=\int_{\Lambda}\left\langle e^{\prime}, \widetilde{f}(x)\right\rangle \mathrm{d} \mu(x)
\end{aligned}
$$

holds for every $e^{\prime} \in E^{\prime}$ where we used the scalar Leibniz' rule for differentiation under the integral sign for the first equation which can be applied due to our assumptions (see [7], 5.7 Satz, p. 147-148]). For $e^{\prime} \in E^{\prime}$ there is $N \in \mathcal{N}_{\mu}$ such that for every $x \in X \backslash\left(N \cup N_{0}\right)$ and $n \in \mathbb{N}$ there is $\theta \in[0,1]$ with

$$
\left\langle e^{\prime}, f_{n}(x)\right\rangle=\frac{\left\langle e^{\prime}, f\left(x, t+h_{n} e_{j}\right)\right\rangle-\left\langle e^{\prime}, f(x, t)\right\rangle}{h_{n}}=\left(\partial_{t_{j}}\right)^{\mathbb{K}}\left\langle e^{\prime}, f\left(x, t+\theta h_{n} e_{j}\right)\right\rangle
$$

by the mean value theorem $(\mathbb{K}=\mathbb{R})$ implying

$$
\left|\left\langle e^{\prime}, f_{n}(x)\right\rangle\right|=\left|\left(\partial_{t_{j}}\right)^{\mathbb{K}}\left\langle e^{\prime}, f\left(x, t+\theta h_{n} e_{j}\right)\right\rangle\right| \leq\left|\left\langle e^{\prime}, \psi_{j}(x)\right\rangle\right| \text {. }
$$

Due to $(8)$ for every $\Lambda \in \Sigma$ and $e^{\prime} \in E^{\prime},(9)$ and the quasi-completeness of $E$ we can apply the dominated convergence theorem for the Pettis-integral [19, Theorem 2, p. 162-163] again and obtain that $\widetilde{f}$ is Pettis-integrable on $\Sigma$ plus

$$
\begin{aligned}
\left(\partial_{t_{j}}\right)^{E} g_{\Lambda}(t) & =\lim _{n \rightarrow \infty} \frac{g_{\Lambda}\left(t+h_{n} e_{j}\right)-g_{\Lambda}(t)}{h_{n}}=\lim _{n \rightarrow \infty} \int_{\Lambda} f_{n}(x) \mathrm{d} \mu(x) \\
& =\int_{\Lambda} \tilde{f}(x) \mathrm{d} \mu(x)=\int_{\Lambda}\left(\partial_{t_{j}}\right)^{E} f(x, t) \mathrm{d} \mu(x) .
\end{aligned}
$$

The continuity of $\left(\partial_{t_{j}}\right)^{E} g_{\Lambda}$ follows from Lemma 4.3 by replacing $f$ with $\left(\partial_{t_{j}}\right)^{E} f$. For $\mathbb{K}=\mathbb{C}$ we just have to substitute $\left\langle e^{\prime}, \cdot\right\rangle$ by $\operatorname{Re}\left\langle e^{\prime}, \cdot\right\rangle$ (real part) and $\operatorname{Im}\left\langle e^{\prime}, \cdot\right\rangle$ (imaginary part) in the considerations above.

Now, we are able to define the convolution of a vector-valued and a scalar-valued continuous function via the Pettis-integral, if one of them has compact support, and to show some of its basic properties which are known from the convolution of scalar-valued functions (scalar convolution). For the properties of the scalar convolution see e.g. [23, Chap. 26, p. 278-283].

LEMMA 4.5. Let $E$ be a quasi-complete lcHs, $k, n \in \mathbb{N}_{0, \infty}, f \in \mathcal{C}^{k}\left(\mathbb{R}^{d}, E\right)$ and $g \in \mathcal{C}^{n}\left(\mathbb{R}^{d}\right)$, either one having compact support. The convolution

$$
f * g: \mathbb{R}^{d} \rightarrow E, \quad(f * g)(x):=\int_{\mathbb{R}^{d}} f(y) g(x-y) \mathrm{d} y,
$$

is well-defined, $\operatorname{supp}(f * g) \subset \operatorname{supp} f+\operatorname{supp} g, f * g=g * f$, where

$$
g * f: \mathbb{R}^{d} \rightarrow E, \quad(g * f)(x):=\int_{\mathbb{R}^{d}} g(y) f(x-y) \mathrm{d} y,
$$

and $f * g \in \mathcal{C}^{n}\left(\mathbb{R}^{d}, E\right)$ plus

$$
\begin{aligned}
& \left(\partial^{\beta}\right)^{E}(f * g)=f *\left(\left(\partial^{\beta}\right)^{\mathbb{K}} g\right), \quad|\beta| \leq n, \\
& \left(\partial^{\beta}\right)^{E}(f * g)=\left(\left(\partial^{\beta}\right)^{E} f\right) * g, \quad|\beta| \leq \min (k, n) .
\end{aligned}
$$


Proof. Let $h: \mathbb{R}^{d} \times \mathbb{R}^{d} \rightarrow E, h(y, x):=f(y) g(x-y)$. First, we show that $h(\cdot, x)$ is Pettis-integrable on $\mathscr{L}\left(\mathbb{R}^{d}\right)$ for every $x \in \mathbb{R}^{d}$ implying that $f * g$ is well-defined. We note that $\left\langle e^{\prime}, h(\cdot, x)\right\rangle \in \mathcal{L}^{1}\left(\mathbb{R}^{d}, \lambda\right)$ for every $e^{\prime} \in E^{\prime}$ and $x \in \mathbb{R}^{d}$. Let $x \in \mathbb{R}^{d}$ and $\Lambda \in \mathscr{L}\left(\mathbb{R}^{d}\right)$. We define the linear map

$$
I_{\Lambda, x}: E^{\prime} \rightarrow \mathbb{K}, I_{\Lambda, x}\left(e^{\prime}\right):=\int_{\Lambda}\left\langle e^{\prime}, h(y, x)\right\rangle \mathrm{d} y .
$$

Setting $K_{f}:=\operatorname{supp} f$ and $K_{g}:=\operatorname{supp} g$, we observe that

$$
I_{\Lambda, x}\left(e^{\prime}\right)=\int_{\Lambda \cap K_{f}}\left\langle e^{\prime}, f(y) g(x-y)\right\rangle \mathrm{d} y=\int_{\Lambda \cap\left(x-K_{g}\right)}\left\langle e^{\prime}, f(y) g(x-y)\right\rangle \mathrm{d} y .
$$

If $K_{f}=\operatorname{supp} f$ is compact, we get

$$
\left|I_{\Lambda, x}\left(e^{\prime}\right)\right| \leq \lambda\left(K_{f}\right) \sup \left\{\left|e^{\prime}(z)\right| \mid z \in f\left(K_{f}\right) g\left(x-K_{f}\right)\right\} .
$$

The set $f\left(K_{f}\right) g\left(x-K_{f}\right)$ is compact in $E$ and thus the closure of its absolutely convex hull is compact in $E$ as well by [24, 9-2-10 Example, p. 134] because $E$ is quasi-complete. Hence it follows that $I_{\Lambda, x} \in\left(E_{\kappa}^{\prime}\right)^{\prime} \cong E$ by the theorem of Mackey-Arens meaning that there is $e_{\Lambda}(x) \in E$ such that

$$
\left\langle e^{\prime}, e_{\Lambda}(x)\right\rangle=I_{\Lambda, x}\left(e^{\prime}\right)=\int_{\Lambda}\left\langle e^{\prime}, h(y, x)\right\rangle \mathrm{d} y
$$

for all $e^{\prime} \in E^{\prime}$. Thus $h(\cdot, x)$ is Pettis-integrable on $\mathscr{L}\left(\mathbb{R}^{d}\right)$ and

$$
(f * g)(x)=e_{\mathbb{R}^{d}}(x) \frac{\overline{\overline{5}}}{\sqrt{5}} e_{K_{f}}(x)=e_{x-K_{g}}(x)
$$

for every $x \in \mathbb{R}^{d}$ if $K_{f}=\operatorname{supp} f$ is compact. If $K_{g}=\operatorname{supp} g$ is compact, then the estimate

$$
\left|I_{\Lambda, x}\left(e^{\prime}\right)\right| \leq \lambda\left(x-K_{g}\right) \sup \left\{\left|e^{\prime}(z)\right| \mid z \in f\left(x-K_{g}\right) g\left(K_{g}\right)\right\}
$$

yields to the Pettis-integrability in the same manner.

Let $x \notin \operatorname{supp} f+\operatorname{supp} g$. If $y \notin \operatorname{supp} f$, then $h(y, x)=0$. If $y \in \operatorname{supp} f$, then $x-y \notin$ $\operatorname{supp} g$ and thus $h(y, x)=0$. Hence we have $h(\cdot, x)=0$ implying $\operatorname{supp}(f * g) \subset \operatorname{supp} f+$ supp $g$. From

$$
\begin{aligned}
\left\langle e^{\prime},(f * g)(x)\right\rangle & =\int_{\mathbb{R}^{d}}\left\langle e^{\prime}, f(y) g(x-y)\right\rangle \mathrm{d} y=\int_{\mathbb{R}^{d}}\left\langle e^{\prime}, f(y)\right\rangle g(x-y) \mathrm{d} y \\
& =\left(\left(e^{\prime} \circ f\right) * g\right)(x)=\left(g *\left(e^{\prime} \circ f\right)\right)(x)=\int_{\mathbb{R}^{d}}\left\langle e^{\prime}, g(y) f(x-y)\right\rangle \mathrm{d} y
\end{aligned}
$$

for every $x \in \mathbb{R}^{d}$ and $e^{\prime} \in E^{\prime}$, where we used the commutativity of scalar convolution for the fourth equation, it follows that

$$
(f * g)(x)=e_{\mathbb{R}^{d}}(x)=(g * f)(x)
$$

for every $x \in \mathbb{R}^{d}$.

Next, we show that $f * g \in \mathcal{C}^{n}\left(\mathbb{R}^{d}, E\right)$ and 110 holds by applying Lemma 4.3 and 4.4. So we have to check that the conditions a)-c) of these lemmas are fulfilled. First, fix $x_{0} \in \mathbb{R}^{d}$, let $\varepsilon>0$ and $\beta \in \mathbb{N}_{0}^{d},|\beta| \leq n$. If $K_{f}=\operatorname{supp} f$ is compact, we set $h_{f, \beta}:=$ $\left(\partial_{x}^{\beta}\right)^{E} h_{\mid K_{f} \times \mathbb{B}_{\varepsilon}\left(x_{0}\right)}$ and observe that $h_{\mid K_{f} \times \mathbb{B}_{\varepsilon}\left(x_{0}\right)}(y, \cdot) \in \mathcal{C}^{n}\left(\mathbb{B}_{\varepsilon}\left(x_{0}\right), E\right)$ for every $y \in K_{f}$ 
(condition b)). It follows from the theorem of Mackey-Arens and

$$
\left|\int_{\omega}\left\langle e^{\prime}, h_{f, \beta}(y, x)\right\rangle \mathrm{d} Y\right| \leq \lambda\left(K_{f}\right) \sup \left\{\left|e^{\prime}(z)\right| \mid z \in f\left(K_{f}\right)\left(\partial^{\beta}\right)^{\mathbb{K}} g\left(\overline{\mathbb{B}_{\varepsilon}\left(x_{0}\right)}-K_{f}\right)\right\}
$$

for every $e^{\prime} \in E^{\prime}, \omega \in \mathscr{L}\left(\mathbb{R}^{d}\right)_{\mid K_{f}}$ and $x \in \mathbb{B}_{\varepsilon}\left(x_{0}\right)$ that $h_{f, \beta}(\cdot, x)$ is Pettis-integrable on $\mathscr{L}\left(\mathbb{R}^{d}\right)_{\mid K_{f}}$ for every $x \in \mathbb{B}_{\varepsilon}\left(x_{0}\right)$ (condition a)). Now, we check that condition c) is satisfied. We observe that the estimate

$$
\left|\int_{\omega}\left\langle e^{\prime}, f(y)\right\rangle \mathrm{d} y\right| \leq \lambda\left(K_{f}\right) \sup \left\{\left|e^{\prime}(z)\right| \mid z \in f\left(K_{f}\right)\right\}
$$

for every $e^{\prime} \in E^{\prime}$ and $\omega \in \mathscr{L}\left(\mathbb{R}^{d}\right)_{\mid K_{f}}$ implies that $f_{\mid K_{f}}$ is Pettis-integrable on $\mathscr{L}\left(\mathbb{R}^{d}\right)_{\mid K_{f}}$ due to the theorem of Mackey-Arens again. The inequality

$$
\begin{aligned}
\left|\left\langle e^{\prime}, h_{f, \beta}(y, x)\right\rangle\right| & =\left|\left\langle e^{\prime}, f(y)\left(\partial_{x}^{\beta}\right)^{\mathbb{K}}[x \mapsto g(x-y)]\right\rangle\right| \\
& \leq\left|\left\langle e^{\prime}, f(y)\right\rangle\right| \sup \left\{\left|\left(\partial^{\beta}\right)^{\mathbb{K}} g(z)\right| \mid z \in \overline{\mathbb{B}_{\varepsilon}\left(x_{0}\right)}-K_{f}\right\} \\
& \leq\left|\left\langle e^{\prime}, q_{\overline{\mathbb{B}_{\varepsilon}\left(x_{0}\right)}-K_{f}, n}(g) \cdot f(y)\right\rangle\right|
\end{aligned}
$$

for every $e^{\prime} \in E^{\prime}$ and $(y, x) \in K_{f} \times \mathbb{B}_{\varepsilon}\left(x_{0}\right)$ with the seminorm $q_{\overline{\mathbb{B}_{\varepsilon}\left(x_{0}\right)}-K_{f}, n}$ from (1) yields to condition c) being satisfied. Hence $f * g \in \mathcal{C}^{n}\left(\mathbb{B}_{\varepsilon}\left(x_{0}\right), E\right)$ by Lemma 4.3 if $n=0$ and by Lemma 4.4 if $n=1$ as well as

$$
\begin{aligned}
\partial_{x_{j}}^{E}(f * g)(x) & =\partial_{x_{j}}^{E}\left[x \mapsto \int_{\mathbb{R}^{d}} f(y) g(x-y) \mathrm{d} y\right]=\partial_{x_{j}}^{E}\left[x \mapsto \int_{K_{f}} f(y) g(x-y) \mathrm{d} y\right] \\
& =\int_{K_{f}} f(y)\left(\partial_{x_{j}}\right)^{\mathbb{K}}[x \mapsto g(x-y)] \mathrm{d} y=\int_{\mathbb{R}^{d}} f(y)\left(\partial^{e_{j}}\right)^{\mathbb{K}} g(x-y) \mathrm{d} y \\
& =\left(f *\left(\left(\partial^{e_{j}}\right)^{\mathbb{K}} g\right)\right)(x)
\end{aligned}
$$

for every $x \in \mathbb{B}_{\varepsilon}\left(x_{0}\right)$. Letting $\varepsilon \rightarrow \infty$, we obtain the result for $n=0$ and $n=1$ if $K_{f}=\operatorname{supp} f$ is compact. For $n \geq 2$ it follows from induction on the order $|\beta|$. If $K_{g}=\operatorname{supp} g$ is compact, the same approach with $h_{g, \beta}:=\left(\partial_{x}^{\beta}\right)^{E} h_{\mid K_{g} \times \mathbb{B}_{\varepsilon}\left(x_{0}\right)}$ instead of $h_{f, \beta}$ proves the statement. Furthermore, for $|\beta| \leq \min (k, n)$ we get

$$
\begin{aligned}
& \left\langle e^{\prime},\left(\partial^{\beta}\right)^{E}(f * g)(x)\right\rangle \\
= & \int_{\mathbb{R}^{d}}\left\langle e^{\prime}, f(y)\left(\partial^{\beta}\right)^{\mathbb{K}} g(x-y)\right\rangle \mathrm{d} y=\int_{\mathbb{R}^{d}}\left(e^{\prime} \circ f\right)(y)\left(\partial^{\beta}\right)^{\mathbb{K}} g(x-y) \mathrm{d} y \\
= & \left(\left(e^{\prime} \circ f\right) *\left(\left(\partial^{\beta}\right)^{\mathbb{K}} g\right)\right)(x)=\left(\left(\partial^{\beta}\right)^{\mathbb{K}}\left(e^{\prime} \circ f\right) * g\right)(x) \\
= & \left(\left(e^{\prime} \circ\left(\partial^{\beta}\right)^{E} f\right) * g\right)(x)=\int_{\mathbb{R}^{d}}\left\langle e^{\prime},\left(\partial^{\beta}\right)^{E} f(y) g(x-y)\right\rangle \mathrm{d} y
\end{aligned}
$$

for every $e^{\prime} \in E^{\prime}$ and $x \in \mathbb{R}^{d}$, where we used the corresponding result for the scalar convolution for the fourth equation, implying $\left(\partial^{\beta}\right)^{E}(f * g)=\left(\left(\partial^{\beta}\right)^{E} f\right) * g$.

Looking at the lemma above, we see that it differs a bit from the properties known from the convolution of two scalar-valued functions. It is an open problem whether we actually have $f * g \in \mathcal{C}^{\max (k, n)}\left(\mathbb{R}^{d}, E\right)$ and $(11)$ for $|\beta| \leq k$ under the assumptions of the lemma. But since we only apply the lemma above in the case $n=\infty$, this does not affect us. 
We recall the construction of a mollifier from [23, p. 155-156]. Let

$$
\rho: \mathbb{R}^{d} \rightarrow \mathbb{R}, \quad \rho(x):= \begin{cases}C \exp \left(-1 /\left(1-|x|^{2}\right)\right), & |x|<1, \\ 0, & |x| \geq 1,\end{cases}
$$

where $C:=\left(\int_{\mathbb{B}_{1}(0)} \exp \left(-\frac{1}{1-|x|^{2}}\right) \mathrm{d} x\right)^{-1}$. For $n \in \mathbb{N}$ we define the mollifier $\rho_{n}$ given by $\rho_{n}(x):=n^{d} \rho(n x), x \in \mathbb{R}^{d}$. Then we have $\rho_{n} \in \mathcal{C}_{c}^{\infty}\left(\mathbb{R}^{d}\right), \rho_{n} \geq 0$, supp $\rho_{n}=\overline{\mathbb{B}_{1 / n}(0)}$ and $\int_{\mathbb{R}^{d}} \rho_{n}(x) \mathrm{d} x=1$.

We can extend a function $f \in \mathcal{C}_{c}^{k}(\Omega, E), k \in \mathbb{N}_{0, \infty}$ and $\Omega \subset \mathbb{R}^{d}$, to a function $f_{\text {ex }} \in \mathcal{C}_{c}^{k}\left(\mathbb{R}^{d}, E\right)$ by setting $f_{\text {ex }}:=f$ on $\Omega$ and $f_{\text {ex }}:=0$ on $\mathbb{R}^{d} \backslash \Omega$. In this way the convolution $f * g:=\left(f_{\text {ex }} * g\right) \mid \Omega$ with a function $g \in \mathcal{C}\left(\mathbb{R}^{d}\right)$ is a well-defined function on $\Omega$ if $E$ is quasi-complete, and we have the following approximation by regularisation in analogy to the scalar-valued case (see e.g. [23, Chap. 15, Corollary 1, p. 158]).

Lemma 4.6. Let $E$ be a quasi-complete lcHs, $k \in \mathbb{N}_{0, \infty}, \mathcal{V}^{k}$ be a family of locally bounded weights on an open set $\Omega \subset \mathbb{R}^{d}$ and $f \in \mathcal{C}_{c}^{k}(\Omega, E)$. Then $\left(f * \rho_{n}\right)$ converges to $f$ in $\mathcal{C} \mathcal{V}_{0}^{k}(\Omega, E)$ as $n \rightarrow \infty$.

Proof. Due to Lemma 4.5 we obtain that $f_{\mathrm{ex}} * \rho_{n} \in \mathcal{C}_{c}^{\infty}\left(\mathbb{R}^{d}, E\right)$ for every $n \in \mathbb{N}$. Since $\mathcal{V}^{k}$ is locally bounded on $\Omega$, we derive $f * \rho_{n} \in \mathcal{C} \mathcal{V}_{0}^{k}(\Omega, E)$. Let $\varepsilon>0, j \in J, l \in\langle k\rangle$ and $\alpha \in \mathfrak{A}$. For $\beta \in \mathbb{N}_{0}^{d},|\beta| \leq l$, there is $\delta_{\beta}>0$ such that for all $x \in \Omega$ and $y \in \mathbb{R}^{d}$ with $|y|=|(x-y)-x| \leq \delta_{\beta}$ we have

$$
p_{\alpha}\left(\left(\partial^{\beta}\right)^{E} f_{\mathrm{ex}}(x-y)-\left(\partial^{\beta}\right)^{E} f(x)\right)<\varepsilon
$$

because the function $\left(\partial^{\beta}\right)^{E} f_{\mathrm{ex}}$ is uniformly continuous on whole $\mathbb{R}^{d}$ as it is continuous with compact support. Therefore we deduce for all $n>1 / \delta_{\beta}$ that $\operatorname{supp} \rho_{n}=\overline{\mathbb{B}_{1 / n}(0)} \subset \overline{\mathbb{B}_{\delta_{\beta}}(0)}$ and hence

$$
\begin{aligned}
& p_{\alpha}\left(\left(\partial^{\beta}\right)^{E}\left(f * \rho_{n}-f\right)(x)\right) \\
= & p_{\alpha}\left(\left(\left(\partial^{\beta}\right)^{E} f\right) * \rho_{n}(x)-\left(\partial^{\beta}\right)^{E} f(x)\right) \\
= & p_{\alpha}\left(\rho_{n} *\left(\left(\partial^{\beta}\right)^{E} f\right)(x)-\left(\partial^{\beta}\right)^{E} f(x)\right) \\
= & p_{\alpha}\left(\int_{\mathbb{R}^{d}}\left(\partial^{\beta}\right)^{E} f_{\mathrm{ex}}(x-y) \rho_{n}(y) \mathrm{d} y-\left(\partial^{\beta}\right)^{E} f(x)\right) \\
= & p_{\alpha}\left(\int_{\mathbb{R}^{d}}\left(\partial^{\beta}\right)^{E} f_{\mathrm{ex}}(x-y) \rho_{n}(y)-\left(\partial^{\beta}\right)^{E} f(x) \rho_{n}(y) \mathrm{d} y\right) \\
= & p_{\alpha}\left(\int_{\overline{\mathbb{B}_{1 / n}(0)}}\left(\partial^{\beta}\right)^{E} f_{\mathrm{ex}}(x-y) \rho_{n}(y)-\left(\partial^{\beta}\right)^{E} f(x) \rho_{n}(y) \mathrm{d} y\right) \\
\leq & \varepsilon \int_{\mathbb{R}^{d}} \rho_{n}(y) \mathrm{d} y=\varepsilon \\
&
\end{aligned}
$$

by Lemma 4.5 for every $x \in \Omega$. As $0 \in \operatorname{supp} \rho_{n}$, we get

$$
\operatorname{supp}\left(\partial^{\beta}\right)^{E}\left(f * \rho_{n}-f\right) \subset\left(\operatorname{supp} f+\operatorname{supp} \rho_{n}\right)=\left(\operatorname{supp} f+\overline{\mathbb{B}_{1 / n}(0)}\right)
$$

for every $|\beta| \leq l$ and $n \in \mathbb{N}$ by virtue of Lemma 4.5. Since supp $f \subset \Omega$ is compact and $\Omega$ open, there is $r>0$ such that $\left(\operatorname{supp} f+\overline{\mathbb{B}_{r}(0)}\right) \subset \Omega$ yielding

$$
\operatorname{supp}\left(\partial^{\beta}\right)^{E}\left(f * \rho_{n}-f\right) \subset\left(\operatorname{supp} f+\overline{\mathbb{B}_{r}(0)}\right)=: K
$$


for all $n \geq 1 / r$. Choosing $\delta:=\min \left\{\delta_{\beta}\left|\beta \in \mathbb{N}_{0}^{d},\right| \beta \mid \leq l\right\}>0$, we obtain for all $n>\max \{1 / \delta, 1 / r\}$ that

$$
\left|f * \rho_{n}-f\right|_{j, l, \alpha}=\sup _{\substack{x \in K \\ \beta \in \mathbb{N}_{0}^{d},|\beta| \leq l}} p_{\alpha}\left(\left(\partial^{\beta}\right)^{E}\left(f * \rho_{n}-f\right)(x)\right) \nu_{j, l}(x) \leq \varepsilon \sup _{x \in K} \nu_{j, l}(x)
$$

which implies our statement since $\mathcal{V}^{k}$ is locally bounded on $\Omega$ and $K \subset \Omega$ is compact.

5. Approximation property. Finally, we dedicate our last section to our main theorem. We start with the case $k=0$.

Proposition 5.1. Let $E$ be an $l c H s$ and $\mathcal{V}^{0}$ a family of locally bounded weights which is locally bounded away from zero on a locally compact Hausdorff space $\Omega$. Then the following statements hold.

a) $\mathcal{C}_{c}^{0}(\Omega) \otimes E$ is dense in $\mathcal{C V}_{0}^{0}(\Omega, E)$.

b) For any $f \in \mathcal{C}_{c}^{0}(\Omega, E)$ and any open neighbourhood $V$ of $\operatorname{supp} f$, for every $\varepsilon>0$, $j \in J$ and $\alpha \in \mathfrak{A}$, there is $g \in \mathcal{C}_{c}^{0}(\Omega) \otimes E$ such that supp $g \subset V$ and $|f-g|_{j, 0, \alpha} \leq \varepsilon$.

c) If $E$ is complete, then

$$
\mathcal{C} \mathcal{V}_{0}^{0}(\Omega, E) \cong \mathcal{C} \mathcal{V}_{0}^{0}(\Omega) \varepsilon E \cong \mathcal{C} \mathcal{V}_{0}^{0}(\Omega) \widehat{\otimes}_{\varepsilon} E
$$

d) $\mathcal{C V}_{0}^{0}(\Omega)$ has the approximation property.

Proof. First, we consider part a). Due to Corollary 3.16 a) and Remark $3.9 \mathcal{C}_{c}^{0}(\Omega) \otimes E$ can be identified with a subspace of $\mathcal{C V}_{0}^{0}(\Omega, E)$ equipped with the induced topology since $\mathcal{V}^{0}$ is locally bounded and locally bounded away from zero.

Let $f \in \mathcal{C V}_{0}^{0}(\Omega, E), \varepsilon>0, j \in J$ and $\alpha \in \mathfrak{A}$ and fix the notation $\nu_{j}:=\nu_{j, 0}$. Then there is a compact set $\widetilde{K} \subset \Omega$ such that

$$
|f|_{\Omega \backslash \widetilde{K}, j, 0, \alpha}=\sup _{x \in \Omega \backslash \widetilde{K}} p_{\alpha}(f(x)) \nu_{j}(x)<\varepsilon .
$$

Let $K:=\widetilde{K}$. Since $\Omega$ is locally compact, every $w \in K$ has an open, relatively compact neighbourhood $U_{w} \subset \Omega$. As $K$ is compact and $K \subset \bigcup_{w \in K} U_{w}$, there are $m \in \mathbb{N}$ and $w_{i} \in K, 1 \leq i \leq m$, such that

$$
K \subset \bigcup_{i=1}^{m} U_{w_{i}}=: W \subset \Omega .
$$

The set $W$ is open and relatively compact because it is a finite union of open, relatively compact sets. The local boundedness of $\mathcal{V}^{0}$ and relative compactness of $W$ imply that

$$
N:=1+\sup _{x \in \bar{W}} \nu_{j}(x)<\infty .
$$

For $x \in K$ we define $V_{x}:=\left\{y \in \Omega \mid p_{\alpha}(f(y)-f(x))<\frac{\varepsilon}{N}\right\}$. Then $V_{x}=f^{-1}\left(B_{\alpha}\left(f(x), \frac{\varepsilon}{N}\right)\right)$, where $B_{\alpha}\left(f(x), \frac{\varepsilon}{N}\right):=\left\{e \in E \mid p_{\alpha}(e-f(x))<\frac{\varepsilon}{N}\right\}$, implying that $V_{x}$ is open in $\Omega$ since $f$ is continuous. Hence we get $K \subset \bigcup_{x \in K} V_{x}$ and conclude that there are $n \in \mathbb{N}$ and $x_{i} \in K, 1 \leq i \leq n$, such that $K \subset \bigcup_{i=1}^{n} V_{x_{i}}$ from the compactness of $K$. We note that

$$
K=(K \cap \bar{W}) \subset \bigcup_{i=1}^{n}\left(V_{x_{i}} \cap \bar{W}\right) .
$$


The sets $V_{x_{i}} \cap \bar{W}$ are open in the compact Hausdorff space $\bar{W}$ with respect to the topology induced by $\Omega$. Since the compact Hausdorff space $\bar{W}$ is normal by [4, Chap. IX, $\S 4.1$, Proposition 1, p. 181] and $K$ is closed in $\bar{W}$, there is a family of non-negative real-valued continuous functions $\left(\varphi_{i}\right)$ with $\operatorname{supp} \varphi_{i} \subset\left(V_{x_{i}} \cap \bar{W}\right)$ such that $\sum_{i=1}^{n} \varphi_{i}=1$ on $K$ and $\sum_{i=1}^{n} \varphi_{i} \leq 1$ on $\bar{W}$ by [4, Chap. IX, $\S 4.3$, Corollary, p. 186]. By trivially extending $\varphi_{i}$ on $\Omega \backslash \bar{W}$, we obtain $\varphi_{i} \in \mathcal{C}_{c}^{0}(\Omega)$ because $\bar{W}$ is compact. We define

$$
g:=\sum_{i=1}^{n} \varphi_{i} \otimes f\left(x_{i}\right) \in \mathcal{C}_{c}^{0}(\Omega) \otimes E
$$

and observe supp $g \subset \bigcup_{i=1}^{n}\left(V_{x_{i}} \cap \bar{W}\right)$. If $x \in K$, then $\varphi_{i}(x) p_{\alpha}\left(f(x)-f\left(x_{i}\right)\right)=0$ if $x \notin V_{x_{i}} \cap \bar{W}$, and

$$
\begin{aligned}
p_{\alpha}(f(x)-g(x)) & =p_{\alpha}\left(\sum_{i=1}^{n} \varphi_{i}(x)\left(f(x)-f\left(x_{i}\right)\right)\right) \leq \sum_{i=1}^{n} \varphi_{i}(x) p_{\alpha}\left(f(x)-f\left(x_{i}\right)\right) \\
& \leq \sum_{i=1}^{n} \varphi_{i}(x) \frac{\varepsilon}{N}=\frac{\varepsilon}{N}
\end{aligned}
$$

yielding to

$$
\sup _{x \in K} p_{\alpha}((f-g)(x)) \nu_{j}(x) \leq \sup _{x \in K} \frac{\varepsilon}{N} \nu_{j}(x) \leq \sup _{x \in \bar{W}} \frac{\varepsilon}{N} \nu_{j}(x)=\frac{\varepsilon}{N} \cdot(N-1)<\varepsilon .
$$

If $x \notin K$, then $\varphi_{i}(x) f\left(x_{i}\right)=0$ if $x \notin\left(V_{x_{i}} \cap \bar{W}\right) \backslash K$. If $x \in\left(V_{x_{i}} \cap \bar{W}\right) \backslash K$, then

$$
p_{\alpha}\left(\varphi_{i}(x) f\left(x_{i}\right)\right) \leq \varphi_{i}(x)\left(p_{\alpha}\left(f\left(x_{i}\right)-f(x)\right)+p_{\alpha}(f(x))\right) \leq \varphi_{i}(x)\left(\frac{\varepsilon}{N}+p_{\alpha}(f(x))\right)
$$

yielding to

$$
\begin{aligned}
& |f-g|_{\Omega \backslash K, j, 0, \alpha} \\
= & \sup _{x \in \Omega \backslash K} p_{\alpha}((f-g)(x)) \nu_{j}(x) \leq \sup _{x \in \Omega \backslash K}\left(p_{\alpha}(f(x))+p_{\alpha}(g(x))\right) \nu_{j}(x) \\
\leq & \varepsilon+\sup _{x \in \Omega \backslash K} \sum_{i=1}^{n} p_{\alpha}\left(\varphi_{i}(x) f\left(x_{i}\right)\right) \nu_{j}(x) \leq \varepsilon+\sup _{x \in \Omega \backslash K} \sum_{i=1}^{n} \varphi_{i}(x)\left(\frac{\varepsilon}{N}+p_{\alpha}(f(x))\right) \nu_{j}(x) \\
\leq & 2 \varepsilon+\frac{\varepsilon}{N} \sup _{x \in \Omega \backslash K} \sum_{i=1}^{n} \varphi_{i}(x) \nu_{j}(x) \leq 2 \varepsilon+\frac{\varepsilon}{N} \sup _{x \in \bar{W}} \sum_{i=1}^{n} \varphi_{i}(x) \nu_{j}(x) \\
\leq & 2 \varepsilon+\frac{\varepsilon}{N} \cdot(N-1)<3 \varepsilon
\end{aligned}
$$

implying

$$
|f-g|_{j, 0, \alpha}<4 \varepsilon
$$

which proves part a).

Part c) follows from a) and Corollary 3.16 b) because $\mathcal{C} \mathcal{V}_{0}^{0}(\Omega)$ is complete by Proposition 3.7 Part d) is implied by part c). Let us turn to part b). Let $f \in \mathcal{C}_{c}^{0}(\Omega, E)$ and $V$ be an open neighbourhood of $\widetilde{K}:=\operatorname{supp} f$. Then we can replace $(13)$ by

$$
K=(K \cap V \cap \bar{W}) \subset \bigcup_{i=1}^{n}\left(V_{x_{i}} \cap V \cap \bar{W}\right)
$$


and then the open sets $V_{x_{i}}$ by the open sets $V_{x_{i}} \cap V$ in what follows (13). This gives

$$
\operatorname{supp} g \subset\left(\bigcup_{i=1}^{n}\left(V_{x_{i}} \cap V \cap \bar{W}\right)\right) \subset V
$$

proving b).

If $\Omega$ is an open subset of $\mathbb{R}^{d}$, we can choose a smooth partition of unity (see e.g. [12, Theorem 1.4.5, p. 28]) and even deduce that $\mathcal{C}_{c}^{\infty}(\Omega) \otimes E$ is dense in $\mathcal{C V}_{0}^{0}(\Omega, E)$ under the assumptions of the proposition above.

The proof of part a) is a modification of the proof of [2, 5.1 Satz, p. 204] by Bierstedt. Since $\Omega$ is locally compact and not just a completely regular Hausdorff space, we can use the partition of unity from [4, Chap. IX, §4.1, Proposition 1, p. 181]. Bierstedt has to use the partition of unity from [20, 23, Lemma 2, p. 71] and due to the assumptions of this lemma he cannot choose $K=\widetilde{K}$ but has to use

$$
K^{\prime}:=\left\{x \in \Omega \mid p_{\alpha}(f(x)) \nu_{j}(x) \geq \varepsilon\right\} \subset \widetilde{K} .
$$

Bierstedt's assumption that $\nu_{j}$ is upper semi-continuous guarantees that $K^{\prime}$ is closed and thus compact as a closed subset of the compact set $\widetilde{K}$. Choosing $K:=K^{\prime}$, the proof above works as well where the existence of the open set $W \subset \Omega$ is a consequence of the upper semi-continuity of $\nu_{j}$ again. Comparing Theorem 1.1 and Proposition 5.1, we see that Theorem 1.1 is far more general concerning the spaces $\Omega$ involved but the condition of $\mathcal{V}^{0}$ being a locally bounded family in Proposition 5.1 is weaker than the condition of being a family of upper semi-continuous weights in Theorem 1.1 Let us phrase our main theorem.

THEOREM 5.2. Let $E$ be an lcHs, $k \in \mathbb{N}_{\infty}$ and $\mathcal{V}^{k}$ be a family of locally bounded weights which is locally bounded away from zero on an open set $\Omega \subset \mathbb{R}^{d}$. Let $\mathcal{C} \mathcal{V}_{0}^{k}(\Omega)$ be barrelled and $\mathcal{C}_{c}^{k}(\Omega, E)$ dense in $\mathcal{C V}_{0}^{k}(\Omega, E)$. Then the following statements hold.

a) $\mathcal{C}_{c}^{\infty}(\Omega) \otimes E$ is dense in $\mathcal{C V}_{0}^{k}(\Omega, E)$.

b) If $E$ is complete, then

$$
\mathcal{C} \mathcal{V}_{0}^{k}(\Omega, E) \cong \mathcal{C} \mathcal{V}_{0}^{k}(\Omega) \varepsilon E \cong \mathcal{C} \mathcal{V}_{0}^{k}(\Omega) \widehat{\otimes}_{\varepsilon} E
$$

c) $\mathcal{C V}_{0}^{k}(\Omega)$ has the approximation property.

Proof. It suffices to prove part a) because part b) follows from a) and Corollary 3.16 b) since $\mathcal{C V}_{0}^{k}(\Omega)$ is complete by Proposition 3.7. Then part c) is a consequence of b). Let us turn to part a). Since $\mathcal{C} \mathcal{V}_{0}^{k}(\Omega)$ is barrelled, $\mathcal{V}^{k}$ locally bounded and locally bounded away from zero, the space $\mathcal{C}_{c}^{\infty}(\Omega) \otimes E$ can be considered as a topological subspace of $\mathcal{C V}_{0}^{k}(\Omega) \otimes_{\varepsilon} E$ by Corollary 3.16 a) and Remark 3.9 when equipped with the induced topology.

Let $f \in \mathcal{C V}_{0}^{k}(\Omega, E), \varepsilon>0, j \in J, l \in\langle k\rangle$ and $\alpha \in \widehat{\mathfrak{A}}$ where $\left(p_{\alpha}\right)_{\alpha \in \widehat{\mathfrak{A}}}$ is the system of seminorms describing the locally convex topology of the completion $\widehat{E}$ of $E$. In the following we consider functions with values in $E$ also as functions with values in $\widehat{E}$ and note that $\mathcal{C} \mathcal{V}_{0}^{k}(\Omega, \widehat{E})$ is the completion of $\mathcal{C} \mathcal{V}_{0}^{k}(\Omega, E)$ by Proposition 3.7. Thus the topologies of $\mathcal{C} \mathcal{V}_{0}^{k}(\Omega, E)$ and $\mathcal{C} \mathcal{V}_{0}^{k}(\Omega, \widehat{E})$ coincide on $\mathcal{C} \mathcal{V}_{0}^{k}(\Omega, E)$. The density of $\mathcal{C}_{c}^{k}(\Omega, E)$ in $\mathcal{C} \mathcal{V}_{0}^{k}(\Omega, E)$ yields that there is $\widetilde{f} \in \mathcal{C}_{c}^{k}(\Omega, E)$ such that $|f-\widetilde{f}|_{j, l, \alpha}<\varepsilon / 3$. Further, there 
is $N_{0} \in \mathbb{N}$ with $\left|\tilde{f}-\tilde{f} * \rho_{n}\right|_{j, l, \alpha}<\varepsilon / 3$ for all $n \geq N_{0}$ by Lemma 4.6 as $\widehat{E}$ is complete. Let $K_{1}:=\operatorname{supp} \widetilde{f}$ and choose an open neighbourhood $V$ of $K_{1}$ such that $V$ is relatively compact in $\Omega$ which is possible since $K_{1}$ is compact and $\Omega \subset \mathbb{R}^{d}$ open. Since $\mathcal{V}^{k}$ is locally bounded away from zero, there is $i \in J$ such that

$$
C_{1}:=\sup _{x \in \bar{V}} \nu_{i, 0}(x)^{-1}=\left(\inf _{x \in \bar{V}} \nu_{i, 0}(x)\right)^{-1}<\infty .
$$

From the relative compactness of $V$ in $\Omega$ it follows that there is $N_{1} \in \mathbb{N}$ such that

$$
\bar{V}+\overline{\mathbb{B}_{1 / n}(0)} \subset \Omega
$$

for all $n \geq N_{1}$. Choosing $N_{2}:=\max \left\{N_{0}, N_{1}\right\}$ and defining the compact set $K_{2}:=$ $\bar{V}+\overline{\mathbb{B}_{1 / N_{2}}(0)} \subset \Omega$, we get that

$$
C_{2}:=\sup _{x \in K_{2}} \nu_{j, l}(x)<\infty
$$

because $\mathcal{V}^{k}$ is locally bounded. Further, we estimate

$$
C_{3}:=\sup _{\beta \in \mathbb{N}_{0}^{d},|\beta| \leq l} \int_{\mathbb{R}^{d}}\left|\partial^{\beta} \rho_{N_{2}}(y)\right| \mathrm{d} y \leq\left(N_{2}\right)^{l} \sup _{\beta \in \mathbb{N}_{0}^{d},|\beta| \leq l} \int_{\mathbb{R}^{d}}\left|\partial^{\beta} \rho(y)\right| \mathrm{d} y<\infty .
$$

By virtue of Proposition 5.1 b) there is $g=\sum_{m=1}^{q} g_{m} \otimes e_{m} \in \mathcal{C}_{c}^{0}(\Omega) \otimes E$ such that supp $g \subset V$ and

$$
|\tilde{f}-g|_{i, 0, \alpha}<\frac{\varepsilon}{3 C_{1} C_{2} C_{3}} .
$$

By Lemma 4.5 we observe that $g * \rho_{N_{2}} \in \mathcal{C}_{c}^{\infty}(\Omega, E)$ with

$$
\operatorname{supp}\left(g * \rho_{N_{2}}\right) \subset \bar{V}+\overline{\mathbb{B}_{1 / N_{2}}(0)}=K_{2} \subset \Omega
$$

and

$$
g * \rho_{N_{2}}=\sum_{m=1}^{q}\left(g_{m} * \rho_{N_{2}}\right) \otimes e_{m} \in \mathcal{C}_{c}^{\infty}(\Omega) \otimes E .
$$

Thus we have by Lemma 4.5

$$
\operatorname{supp}\left(\tilde{f} * \rho_{N_{2}}\right) \subset \bar{V}+\overline{\mathbb{B}_{1 / N_{2}}(0)}=K_{2}
$$

yielding

$$
\operatorname{supp}\left(\tilde{f} * \rho_{N_{2}}-g * \rho_{N_{2}}\right) \subset \bar{V}+\overline{\mathbb{B}_{1 / N_{2}}(0)}=K_{2} \subset \Omega
$$

and

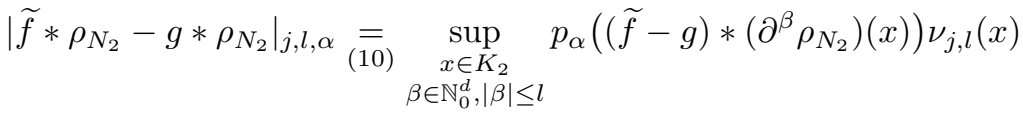

$$
\begin{aligned}
& =\sup _{\substack{x \in K_{2} \\
\beta \in \mathbb{N}_{0}^{d},|\beta| \leq l}} p_{\alpha}\left(\int_{\mathbb{R}^{d}}\left(\partial^{\beta} \rho_{N_{2}}\right)(x-y)\left(\tilde{f}_{\mathrm{ex}}(y)-g_{\mathrm{ex}}(y)\right) \mathrm{d} y\right) \nu_{j, l}(x) \\
& \leq \sup _{\substack{x \in K_{2} \\
\beta \in \mathbb{N}_{0}^{d},|\beta| \leq l}} \int_{\mathbb{R}^{d}}\left|\left(\partial^{\beta} \rho_{N_{2}}\right)(x-y)\right| \mathrm{d} y \sup _{\substack{z \in \operatorname{supp}(\widetilde{f}) \\
\cup \operatorname{supp}(g)}} p_{\alpha}(\tilde{f}(z)-g(z)) \nu_{j, l}(x) \\
& =\sup _{\substack{x \in K_{2} \\
\beta \in \mathbb{N}_{0}^{d},|\beta| \leq l}} \int_{\mathbb{R}^{d}}\left|\left(\partial^{\beta} \rho_{N_{2}}\right)(y)\right| \mathrm{d} y \sup _{z \in \bar{V}} p_{\alpha}(\tilde{f}(z)-g(z)) \nu_{j, l}(x)
\end{aligned}
$$




$$
\begin{aligned}
& \leq C_{3}\left(\sup _{x \in K_{2}} \nu_{j, l}(x)\right)\left(\sup _{z \in \bar{V}} p_{\alpha}(\tilde{f}(z)-g(z))\right) \\
& =C_{3} C_{2} \sup _{z \in \bar{V}} p_{\alpha}(\tilde{f}(z)-g(z)) \nu_{i, 0}(z) \nu_{i, 0}(z)^{-1} \\
& \leq C_{3} C_{2} C_{1}|\tilde{f}-g|_{i, 0, \alpha}<\frac{\varepsilon}{3} .
\end{aligned}
$$

Therefore we deduce

$\left|f-g * \rho_{N_{2}}\right|_{j, l, \alpha} \leq|f-\widetilde{f}|_{j, l, \alpha}+\left|\tilde{f}-\tilde{f} * \rho_{N_{2}}\right|_{j, l, \alpha}+\left|\widetilde{f} * \rho_{N_{2}}-g * \rho_{N_{2}}\right|_{j, l, \alpha}<\frac{\varepsilon}{3}+\frac{\varepsilon}{3}+\frac{\varepsilon}{3}=\varepsilon$.

If we keep in mind that $f \in \mathcal{C V}_{0}^{k}(\Omega, E)$ and $g * \rho_{N_{2}} \in \mathcal{C}_{c}^{\infty}(\Omega) \otimes E$, it follows that $\mathcal{C}_{c}^{\infty}(\Omega) \otimes E$ is dense in $\mathcal{C} \mathcal{V}_{0}^{k}(\Omega, E)$ with respect to the topology of $\mathcal{C} \mathcal{V}_{0}^{k}(\Omega, \widehat{E})$. However, the latter space is just the completion of $\mathcal{C} \mathcal{V}_{0}^{k}(\Omega, E)$ and thus the topologies of $\mathcal{C} \mathcal{V}_{0}^{k}(\Omega, E)$ and $\mathcal{C} \mathcal{V}_{0}^{k}(\Omega, \widehat{E})$ coincide on $\mathcal{C V}_{0}^{k}(\Omega, E)$. Hence $\mathcal{C}_{c}^{\infty}(\Omega) \otimes E$ is dense in $\mathcal{C V}_{0}^{k}(\Omega, E)$.

$\mathcal{C}_{c}^{k}(\Omega, E)$ is dense in $\mathcal{C} \mathcal{V}_{0}^{k}(\Omega, E)$ by Lemma 3.14 if the latter space fulfils the cut-off criterion and the family $\mathcal{V}^{k}$ is locally bounded. $\mathcal{C} \mathcal{V}_{0}^{k}(\Omega)$ is a Fréchet space and thus barrelled by Proposition 3.7 if the $J$ in $\mathcal{V}^{k}=\left(\nu_{j, l}\right)_{j \in J, l \in\langle k\rangle}$ is countable. Let us complement what we said about the standard structure of a family of weights (see the remarks below Definition 3.2 by our additional conditions on the weights collected so far. The standard structure of a (countable) locally bounded family $\mathcal{V}^{k}$ which is bounded away from zero on a locally compact Hausdorff space $\Omega$, resp. on an open set $\Omega \subset \mathbb{R}^{d}$, is given by the following. Let $J:=\mathbb{N},\left(\Omega_{j}\right)_{j \in J}$, be a family of sets such that $\Omega_{j} \subset \Omega_{j+1}$ for all $j \in J$ with $\Omega=\bigcup_{j \in J} \Omega_{j}$ and

$$
\forall K \subset \Omega \text { compact } \exists j \in J: K \subset \Omega_{j} \text {. }
$$

Let $\widetilde{\nu}_{j, l}: \Omega \rightarrow(0, \infty)$ be continuous for all $j \in J, l \in\langle k\rangle$ and increasing in $j \in J$ and in $l \in\langle k\rangle$ such that

$$
\nu_{j, l}(x)=\chi_{\Omega_{j}}(x) \widetilde{\nu}_{j, l}(x), \quad x \in \Omega,
$$

for every $j \in J$ and $l \in\langle k\rangle$ where $\chi_{\Omega_{j}}$ is the indicator function of $\Omega_{j}$. If $\Omega \neq \mathbb{R}^{d}$, then the cut-off criterion may add some restrictions on the structure of the sequence $\left(\Omega_{j}\right)$, e.g. a positive distance from the boundary $\partial \Omega_{j}$ of $\Omega_{j}$ to the boundary $\partial \Omega_{j+1}$ of $\Omega_{j+1}$ for all $j$.

ExAmple 5.3. Let $E$ be an lcHs, $k \in \mathbb{N}_{\infty}$ and $\Omega \subset \mathbb{R}^{d}$ open. Theorem 5.2 can be applied to the following spaces:

a) $\mathcal{C}^{k}(\Omega, E)$ with the topology of uniform convergence of all partial derivatives up to order $k$ on compact subsets of $\Omega$,

b) the Schwartz space $\mathcal{S}\left(\mathbb{R}^{d}, E\right)$,

c) the space $\mathcal{O}_{M}\left(\mathbb{R}^{d}, E\right)$ of multipliers of $\mathcal{S}\left(\mathbb{R}^{d}\right)$,

d) let $\Omega_{j}:=\left\{x=\left(x_{1}, x_{2}\right) \in \mathbb{R}^{2}|1 /(j+1)<| x_{2} \mid<j+1\right\}$ for all $j \in \mathbb{N}$ and

$$
\mathcal{C}_{\exp }^{k}\left(\mathbb{R}^{2} \backslash \mathbb{R}, E\right):=\left\{\left.f \in \mathcal{C}^{k}\left(\mathbb{R}^{2} \backslash \mathbb{R}, E\right)|\forall j \in \mathbb{N}, l \in\langle k\rangle, \alpha \in \mathfrak{A}:| f\right|_{j, l, \alpha}<\infty\right\}
$$

where

$$
|f|_{j, l, \alpha}:=\sup _{\substack{\left(x_{1}, x_{2}\right) \in \Omega_{j} \\ \beta \in \mathbb{N}_{0}^{2},|\beta| \leq l}} p_{\alpha}\left(\left(\partial^{\beta}\right)^{E} f\left(x_{1}, x_{2}\right)\right) e^{-\left|x_{1}\right| /(j+1)} .
$$


Proof. a) From Example 3.5 a) we obtain $\mathcal{C}^{k}(\Omega, E)=\mathcal{C} \mathcal{W}_{0}^{k}(\Omega, E)$ with $\mathcal{W}^{k}:=\left\{\nu_{j, l}:=\right.$ $\left.\chi_{\Omega_{j}} \mid j \in \mathbb{N}, l \in\langle k\rangle\right\}$ where $\left(\Omega_{j}\right)_{j \in \mathbb{N}}$ is a compact exhaustion of $\Omega$. The family of weights $\mathcal{W}^{k}$ is locally bounded and locally bounded away from zero. The Fréchet space $\mathcal{C}^{k}(\Omega)$ is barrelled and the cut-off criterion is fulfilled by Example 3.12 .

b) Due to Example 3.5 b) we have $\mathcal{S}\left(\mathbb{R}^{d}, E\right)=\mathcal{C} \mathcal{V}_{0}^{\infty}\left(\mathbb{R}^{d}, E\right)$ with $\mathcal{V}^{\infty}:=\left\{\nu_{j, l} \mid j \in \mathbb{N}\right.$, $\left.l \in \mathbb{N}_{0}\right\}$ where $\nu_{j, l}(x):=\left(1+|x|^{2}\right)^{l / 2}$ for $x \in \mathbb{R}^{d}$. The family of weights is locally bounded and bounded away from zero, the Fréchet space $\mathcal{S}\left(\mathbb{R}^{d}\right)$ is barrelled and $\mathcal{S}\left(\mathbb{R}^{d}, E\right)$ fulfils the cut-off criterion by Example 3.12

c) The space of multipliers is defined by

$$
\mathcal{O}_{M}\left(\mathbb{R}^{d}, E\right):=\left\{f \in \mathcal{C}^{\infty}\left(\mathbb{R}^{d}, E\right) \mid \forall g \in \mathcal{S}\left(\mathbb{R}^{d}\right), l \in \mathbb{N}_{0}, \alpha \in \mathfrak{A}:\|f\|_{g, l, \alpha}<\infty\right\}
$$

where

$$
\|f\|_{g, l, \alpha}:=\sup _{\substack{x \in \mathbb{R}^{d} \\ \beta \in \mathbb{N}_{0}^{d},|\beta| \leq l}} p_{\alpha}\left(\left(\partial^{\beta}\right)^{E} f(x)\right)|g(x)|
$$

(see [21, $\left.3^{0}\right)$, p. 97]). The space $\mathcal{O}_{M}\left(\mathbb{R}^{d}\right)$ is barrelled by [11, Chap. II, $\S 4, \mathrm{n}^{\circ} 4$, Théorème 16 , p. 131]. Let $J:=\left\{j \subset \mathcal{S}\left(\mathbb{R}^{d}\right) \mid j\right.$ finite $\}$ and define the family $\mathcal{V}^{\infty}$ of weights given by $\nu_{j, l}(x):=\max _{g \in j}|g(x)|, x \in \mathbb{R}^{d}$, for $j \in J$ and $l \in \mathbb{N}_{0}$. It is easily seen that the system of seminorms generated by

$$
|f|_{j, l, \alpha}:=\sup _{\substack{x \in \mathbb{R}^{d} \\ \beta \in \mathbb{N}_{0}^{d},|\beta| \leq l}} p_{\alpha}\left(\left(\partial^{\beta}\right)^{E} f(x)\right) \nu_{j, l}(x), \quad f \in \mathcal{O}_{M}\left(\mathbb{R}^{d}, E\right),
$$

for $j \in J, l \in \mathbb{N}_{0}$ and $\alpha \in \mathfrak{A}$ induces the same topology on $\mathcal{O}_{M}\left(\mathbb{R}^{d}, E\right)$. However, the family $\mathcal{V}^{\infty}$ is directed, locally bounded and bounded away from zero. Further, for every $\varepsilon>0$ there is $r>0$ such that $\left(1+|x|^{2}\right)^{-1}<\varepsilon$ for all $x \notin \overline{\mathbb{B}_{r}(0)}=: K$ which implies for $j \in J$ and $l \in \mathbb{N}_{0}$ that

$$
\nu_{j, l}(x) \leq \varepsilon \max _{g \in j}\left|g(x)\left(1+|x|^{2}\right)\right|=\varepsilon \nu_{i, l}(x), \quad x \notin K,
$$

where $i:=\left\{g \cdot\left(1+|\cdot|^{2}\right) \mid g \in j\right\}$ is a finite subset of $\mathcal{S}\left(\mathbb{R}^{d}\right)$. From Remark 3.4 we conclude that $\mathcal{O}_{M}\left(\mathbb{R}^{d}, E\right)=\mathcal{C} \mathcal{V}^{\infty}\left(\mathbb{R}^{d}, E\right)=\mathcal{C} \mathcal{V}_{0}^{\infty}\left(\mathbb{R}^{d}, E\right)$. Due to Remark 3.11 we note that $\mathcal{O}_{M}\left(\mathbb{R}^{d}, E\right)$ satisfies the cut-off criterion.

d) The family $\mathcal{V}^{k}$ given by $\nu_{j, l}\left(x_{1}, x_{2}\right):=\chi_{\Omega_{j}}\left(x_{1}, x_{2}\right) e^{-\left|x_{1}\right| /(j+1)},\left(x_{1}, x_{2}\right) \in \mathbb{R}^{2} \backslash \mathbb{R}$, for $j \in \mathbb{N}$ and $l \in\langle k\rangle$ is locally bounded and bounded away from zero. For $j \in \mathbb{N}$ and $l \in \mathbb{N}_{0}$ we set $i:=2 j+1, m:=l, \delta:=1 /(2 j+2)$ and for $0<\varepsilon<1$ we choose $K:=\left\{x=\left(x_{1}, x_{2}\right) \in\right.$ $\left.\overline{\Omega_{j}}|| x_{1} \mid \leq-(\ln \varepsilon)(2 j+2)\right\}$. This yields $\mathcal{C}_{\exp }^{k}\left(\mathbb{R}^{2} \backslash \mathbb{R}, E\right)=\mathcal{C} \mathcal{V}^{k}\left(\mathbb{R}^{2} \backslash \mathbb{R}, E\right)=\mathcal{C} \mathcal{V}_{0}^{k}\left(\mathbb{R}^{2} \backslash \mathbb{R}, E\right)$ by Remark 3.4 and that the cut-off criterion is fulfilled. In addition, the Fréchet space $\mathcal{C}_{\exp }^{k}\left(\mathbb{R}^{2} \backslash \mathbb{R}\right)$ is barrelled.

Together with Proposition 5.1 we get from example a) one of our starting points, namely Theorem 1.2 back. Example b) and c) are covered by [21, Proposition 9, p. 108] and [21, Théorème 1, p. 111]. The results b) and c) for the Schwartz space in example b) can also be found in [11, Chap. II, §3, n³, Exemples, p. 80-81] with a different proof using the nuclearity of $\mathcal{S}\left(\mathbb{R}^{d}\right)$. We complete this paper with a comparison of our conditions in Theorem 5.2 with the ones stated by Schwartz in 21] to get the same result for the spaces in example a)-c) but only for $\Omega=\mathbb{R}^{d}$. 
REMARK 5.4. Schwartz treats the case $k>0$ and $\Omega=\mathbb{R}^{d}$ in $[21$. He assumes similar conditions $H_{1}-H_{4}$ for the space $\mathcal{H}^{k}\left(\mathbb{R}^{d}\right):=\mathcal{H}^{k}\left(\mathbb{R}^{d}, \mathbb{K}\right)$ as we do (see [21, p. 97-98]). In $H_{1}$ the members of his family of weights $\Gamma$ are continuous and for every compact set $K \subset \mathbb{R}^{d}$ there is a weight in $\Gamma$ which is non-zero on $K . \mathcal{H}^{k}\left(\mathbb{R}^{d}\right)$ is the space of functions $f \in \mathcal{C}^{k}\left(\mathbb{R}^{d}\right)$ such that $\gamma \partial^{\beta} f$ is bounded on $\mathbb{R}^{d}$ for every $\gamma \in \Gamma$ and $|\beta| \leq k$. This yields to $\mathcal{C}_{c}^{k}\left(\mathbb{R}^{d}\right) \subset \mathcal{H}^{k}\left(\mathbb{R}^{d}\right) \subset \mathcal{C}^{k}\left(\mathbb{R}^{d}\right)$ algebraically. In $H_{2}$ he demands that $\mathcal{H}^{k}\left(\mathbb{R}^{d}\right)$ is a locally convex Hausdorff space and that the inclusions $\mathcal{C}_{c}^{k}\left(\mathbb{R}^{d}\right) \hookrightarrow \mathcal{H}^{k}\left(\mathbb{R}^{d}\right) \hookrightarrow \mathcal{C}^{k}\left(\mathbb{R}^{d}\right)$ are continuous where $\mathcal{C}^{k}\left(\mathbb{R}^{d}\right)$ has its usual topology and $\mathcal{C}_{c}^{k}\left(\mathbb{R}^{d}\right)$ its inductive limit topology. In $H_{3}$ he supposes that a subset $B \subset \mathcal{H}^{k}\left(\mathbb{R}^{d}\right)$ is bounded if and only if for every $\gamma \in \Gamma$ and $|\beta| \leq k$ the set $\left\{\gamma(x) \partial^{\beta} f(x) \mid x \in \mathbb{R}^{d}, f \in B\right\}$ is bounded in $\mathbb{K}$. In $H_{4}$ he assumes that on every bounded subset of $\mathcal{H}^{k}\left(\mathbb{R}^{d}\right)$ the topology of $\mathcal{H}^{k}\left(\mathbb{R}^{d}\right)$ and the induced topology of $\mathcal{C}^{k}\left(\mathbb{R}^{d}\right)$ coincide.

He defines the $E$-valued version $\mathcal{H}^{k}\left(\mathbb{R}^{d}, E\right)$ which corresponds to the space $\mathcal{H}^{k}\left(\mathbb{R}^{d}\right)$ for $\mathcal{H}^{k}=\mathcal{C}_{c}^{k}, \mathcal{C}^{k}, \mathcal{S}$ and $\mathcal{O}_{M}$ and shows that the statements of Theorem 5.2 hold for all of them but $\mathcal{H}^{k}=\mathcal{C}_{c}^{k}$ (see [21, p. 94-97], [21, Proposition 9, p. 108] and [21, Théorème 1, p. 111]).

In comparison, our conditions of local boundedness of $\mathcal{V}^{k}$ and being locally bounded away from zero on $\Omega=\mathbb{R}^{d}$ imply $H_{1}$ and $H_{2}$ if the members of $\mathcal{V}^{k}$ are continuous. The assumption that the members of $\mathcal{V}^{k}$ are continuous is not a big difference if the members of the family $\mathcal{V}^{k}$ have a structure like in (14). Then one may replace the indicator functions $\chi_{\Omega_{j}}$ by a smoothed version, e.g. by convolution of the indicator function with a suitable mollifier, and then one gets a family of continuous weights which generates the same topology. The condition $H_{3}$ is clearly fulfilled for the spaces $\mathcal{C} \mathcal{V}^{k}\left(\mathbb{R}^{d}\right)$ and the topology on them is called 'topologie naturelle' by Schwartz (see [21, p. 98]). The condition $H_{4}$ implies that $\mathcal{C}_{c}^{k}\left(\mathbb{R}^{d}, E\right)$ is dense in $\mathcal{H}^{k}\left(\mathbb{R}^{d}, E\right)$ for $\mathcal{H}^{k}=\mathcal{C}^{k}, \mathcal{S}$ and $\mathcal{O}_{M}$ and quasi-complete $E$ (see [21, p. 106] and [21, Théorème 1, p. 111]). The same follows in our case from local boundedness and the cut-off criterion.

\section{References}

[1] K.-D. Bierstedt, Gewichtete Räume stetiger vektorwertiger Funktionen und das injektive Tensorprodukt, Ph.D. thesis, Johannes-Gutenberg Universität Mainz, Mainz, 1971.

[2] K.-D. Bierstedt, Gewichtete Räume stetiger vektorwertiger Funktionen und das injektive Tensorprodukt. I, J. Reine Angew. Math. 259 (1973), 186-210.

[3] K.-D. Bierstedt, Gewichtete Räume stetiger vektorwertiger Funktionen und das injektive Tensorprodukt. II, J. Reine Angew. Math. 260 (1973), 133-146.

[4] N. Bourbaki, General Topology, Part 2, Elements of Mathematics, Addison-Wesley, Reading, 1966.

[5] H. Buchwalter, Topologies et compactologies, Publ. Dép. Math. (Lyon) 6 (1969), no. 2, 1-74.

[6] A. Defant, K. Floret, Tensor Norms and Operator Ideals, North-Holland Math. Stud. 176, North-Holland, Amsterdam, 1993.

[7] J. Elstrodt, Maß- und Integrationstheorie, 7th ed., Grundwissen Mathematik, Springer, Berlin, 2011.

[8] R. Engelking, General Topology, Sigma Ser. Pure Math. 6, Heldermann, Berlin, 1989. 
[9] M. Fabian, P. Habala, P. Hájek, V. Montesinos, V. Zizler, Banach Space Theory: The Basis for Linear and Nonlinear Analysis, CMS Books Math., Springer, New York, 2011.

[10] K. Floret, J. Wloka, Einführung in die Theorie der lokalkonvexen Räume, Lecture Notes in Math. 56, Springer, Berlin, 1968.

[11] A. Grothendieck, Produits tensoriels topologiques et espaces nucléaires, 4th ed., Mem. Amer. Math. Soc. 16, Amer. Math. Soc., Providence, 1966.

[12] L. Hörmander, The Analysis of Linear Partial Differential Operators I, 2nd ed., Classics Math., Springer, Berlin, 1990.

[13] I. M. James, Topologies and Uniformities, Springer Undergrad. Math. Ser., Springer, London, 1999.

[14] H. Jarchow, Locally Convex Spaces, Math. Leitfäden, Teubner, Stuttgart, 1981.

[15] W. Kaballo, Aufbaukurs Funktionalanalysis und Operatortheorie, Springer, Berlin, 2014.

[16] A. Kriegl, P. W. Michor, The Convenient Setting of Global Analysis, Math. Surveys Monogr. 53, AMS, Providence, 1997.

[17] K. Kruse, Weighted vector-valued functions and the $\varepsilon$-product, arXiv:1712.01613v6.

[18] R. Meise, D. Vogt, Introduction to Functional Analysis, Oxford Grad. Texts Math. 2, Clarendon Press, Oxford, 1997.

[19] K. Musiał, Vitali and Lebesgue convergence theorems for Pettis integral in locally convex spaces, Atti Sem. Mat. Fis. Univ. Modena 35 (1987), 159-166.

[20] L. Nachbin, Elements of Approximation Theory, Notas de Matemática 33, Instituto de Matemática Pura e Aplicada, Rio de Janeiro, 1965.

[21] L. Schwartz, Espaces de fonctions différentiables à valeurs vectorielles, J. Analyse Math. 4 (1955), 88-148.

[22] L. Schwartz, Théorie des distributions à valeurs vectorielles. I, Ann. Inst. Fourier (Grenoble) 7 (1957), 1-142.

[23] F. Trèves, Topological Vector Spaces, Distributions and Kernels, Dover, Mineola, NY, 2006.

[24] A. Wilansky, Modern Methods in Topological Vector Spaces, McGraw-Hill, New York, 1978. 